\title{
On solutions of a system of variational inequalities and fixed point problems in Banach spaces
}

\author{
Lu-Chuan Ceng ${ }^{1}$, Abdul Latif2 ${ }^{*}$ and Jen-Chih $\mathrm{YaO}^{2,3}$
}

"Correspondence: alatif@kau.edu.sa ${ }^{2}$ Department of Mathematics, King Abdulaziz University, P.O. Box 80203, Jeddah, 21589, Saudi Arabia Full list of author information is available at the end of the article

\begin{abstract}
In this paper, considering the problem of solving a system of variational inequalities and a common fixed point problem of an infinite family of nonexpansive mappings in Banach spaces, we propose a two-step relaxed extragradient method which is based on Korpelevich's extragradient method and viscosity approximation method. Strong convergence results are established.
\end{abstract}

MSC: 49J30; 47H09; 47J20

Keywords: two-step relaxed extragradient method; system of variational inequalities; nonexpansive mapping; sunny nonexpansive retraction; fixed point; uniform smoothness; uniform convexity

\section{Introduction}

In the last three decades, the theory of variational inequalities has been used as a tool to study the Nash equilibrium problem for a finite or infinite number of players; see, for example, [1-6] and the references therein. There are two ways to study the Nash equilibrium problem by using variational inequality technique: (1) system of variational inequalities; (2) variational inequalities defined over the product of sets. If the number of players is finite, then the system of variational inequalities is equivalent to the variational inequality defined over the product of sets; see, for example, $[7,8]$ and the references therein.

Very recently, Cai and $\mathrm{Bu}[9]$ considered the following system of two variational inequalities in the setting of Banach spaces.

Let $C$ be a nonempty, closed and convex subset of a real Banach space $X$, let $B_{1}, B_{2}: C \rightarrow$ $X$ be two nonlinear mappings and $\mu_{1}$ and $\mu_{2}$ be two positive constants. The problem of system of variational inequalities (SVI) [9] is to find $\left(x^{*}, y^{*}\right) \in C \times C$ such that

$$
\begin{cases}\left\langle\mu_{1} B_{1} y^{*}+x^{*}-y^{*}, J\left(x-x^{*}\right)\right\rangle \geq 0, & \forall x \in C, \\ \left\langle\mu_{2} B_{2} x^{*}+y^{*}-x^{*}, J\left(x-y^{*}\right)\right\rangle \geq 0, & \forall x \in C,\end{cases}
$$

where $J$ is the normalized duality mapping. The set of solutions of GSVI (1.1) is denoted by $\operatorname{GSVI}\left(C, B_{1}, B_{2}\right)$. This system could be useful to study the Nash equilibrium problem for two players. They proposed an iterative scheme to compute the approximate solutions of such a system.

@ 2013 Ceng et al.; licensee Springer. This is an Open Access article distributed under the terms of the Creative Commons Attribution License (http://creativecommons.org/licenses/by/2.0), which permits unrestricted use, distribution, and reproduction in any medium, provided the original work is properly cited. 
In particular, if $X=H$, a real Hilbert space, then GSVI (1.1) reduces to the following problem of a system of variational inequalities of finding $\left(x^{*}, y^{*}\right) \in C \times C$ such that

$$
\begin{cases}\left\langle\mu_{1} B_{1} y^{*}+x^{*}-y^{*}, x-x^{*}\right\rangle \geq 0, & \forall x \in C, \\ \left\langle\mu_{2} B_{2} x^{*}+y^{*}-x^{*}, x-y^{*}\right\rangle \geq 0, & \forall x \in C,\end{cases}
$$

where $\mu_{1}$ and $\mu_{2}$ are two positive constants. The set of solutions of problem (1.2) is still denoted by $\operatorname{GSVI}\left(C, B_{1}, B_{2}\right)$.

In this paper, we introduce two-step relaxed extragradient method for solving SVI (1.1) and the common fixed point problem of an infinite family $\left\{S_{n}\right\}$ of nonexpansive mappings of $C$ into itself. Here, the two-step relaxed extragradient method is based on Korpelevich's extragradient method [10] and viscosity approximation method. We first suggest and analyze an implicit iterative algorithm by the two-step relaxed extragradient method in a uniformly convex and 2-uniformly smooth Banach space $X$, and then another explicit iterative algorithm in a uniformly convex Banach space $X$ with a uniformly Gâteaux differentiable norm. On the other hand, we also propose and analyze a composite explicit iterative algorithm by the two-step relaxed extragradient method for solving SVI (1.1) and the common fixed point problem of $\left\{S_{n}\right\}$ in a uniformly convex and 2-uniformly smooth Banach space. The results presented in this paper improve, extend, supplement and develop the corresponding results that have appeared very recently in the literature.

\section{Preliminaries}

Let $X^{*}$ be the dual of $X$. The normalized duality mapping $J: X \rightarrow 2^{X^{*}}$ is defined by

$$
J(x)=\left\{x^{*} \in X^{*}:\left\langle x, x^{*}\right\rangle=\|x\|^{2}=\left\|x^{*}\right\|^{2}\right\}, \quad \forall x \in X,
$$

where $\langle\cdot, \cdot\rangle$ denotes the generalized duality pairing.

Let $C$ be a nonempty closed convex subset of a real Banach space $X$. A mapping $A: C \rightarrow$ $X$ is said to be accretive if for each $x, y \in C$ there exists $j(x-y) \in J(x-y)$ such that

$$
\langle A x-A y, j(x-y)\rangle \geq 0
$$

where $J$ is the normalized duality mapping. $A$ is said to be $\alpha$-strongly accretive if for each $x, y \in C$ there exists $j(x-y) \in J(x-y)$ such that

$$
\langle A x-A y, j(x-y)| \geq \alpha\|x-y\|^{2}
$$

for some $\alpha \in(0,1)$. It is said to be $\beta$-inverse-strongly-accretive if for each $x, y \in C$ there exists $j(x-y) \in J(x-y)$ such that

$$
\langle A x-A y, j(x-y)\rangle \geq \beta\|A x-A y\|^{2}
$$

for some $\beta>0$; and finally $A$ is said to be $\lambda$-strictly pseudocontractive if for each $x, y \in C$ there exists $j(x-y) \in J(x-y)$ such that

$$
\langle A x-A y, j(x-y)\rangle \leq\|x-y\|^{2}-\lambda\|x-y-(A x-A y)\|^{2}
$$

for some $\lambda \in(0,1)$. 
Let $D$ be a subset of $C$ and let $\Pi$ be a mapping of $C$ into $D$. Then $\Pi$ is said to be sunny if

$$
\Pi[\Pi(x)+t(x-\Pi(x))]=\Pi(x)
$$

whenever $\Pi(x)+t(x-\Pi(x)) \in C$ for $x \in C$ and $t \geq 0$. A mapping $\Pi$ of $C$ into itself is called a retraction if $\Pi^{2}=\Pi$. If a mapping $\Pi$ of $C$ into itself is a retraction, then $\Pi(z)=z$ for every $z \in R(\Pi)$, where $R(\Pi)$ is the range of $\Pi$. A subset $D$ of $C$ is called a sunny nonexpansive retract of $C$ if there exists a sunny nonexpansive retraction from $C$ onto $D$.

It is well known that if $X=H$, a Hilbert space, then a sunny nonexpansive retraction $\Pi_{C}$ is coincident with the metric projection from $X$ onto $C$; that is, $\Pi_{C}=P_{C}$. If $C$ is a nonempty closed convex subset of a strictly convex and uniformly smooth Banach space $X$ and if $T: C \rightarrow C$ is a nonexpansive mapping with the fixed point set $\operatorname{Fix}(T) \neq \emptyset$, then the set $\operatorname{Fix}(T)$ is a sunny nonexpansive retract of $C$.

The following lemma concerns the sunny nonexpansive retraction.

Lemma 2.1 (see [11]) Let $C$ be a nonempty closed convex subset of a real smooth Banach space $X$. Let $D$ be a nonempty subset of $C$. Let $\Pi$ be a retraction of $C$ onto $D$ and let $J$ be a normalized duality mapping on $X$. Then the following are equivalent:

(i) $\Pi$ is sunny and nonexpansive;

(ii) $\|\Pi(x)-\Pi(y)\|^{2} \leq\langle x-y, J(\Pi(x)-\Pi(y))\rangle, \forall x, y \in C$;

(iii) $\langle x-\Pi(x), J(y-\Pi(x))\rangle \leq 0, \forall x \in C, y \in D$.

Next, we present some more lemmas which are crucial for the proofs of our results.

Lemma 2.2 (see [12]) Let $\left\{s_{n}\right\}$ be a sequence of nonnegative real numbers satisfying

$$
s_{n+1} \leq\left(1-\alpha_{n}\right) s_{n}+\alpha_{n} \beta_{n}+\gamma_{n}, \quad \forall n \geq 0,
$$

where $\left\{\alpha_{n}\right\},\left\{\beta_{n}\right\}$ and $\left\{\gamma_{n}\right\}$ satisfy the conditions:

(i) $\left\{\alpha_{n}\right\} \subset[0,1]$ and $\sum_{n=0}^{\infty} \alpha_{n}=\infty$;

(ii) $\lim \sup _{n \rightarrow \infty} \beta_{n} \leq 0$;

(iii) $\gamma_{n} \geq 0, \forall n \geq 0$, and $\sum_{n=0}^{\infty} \gamma_{n}<\infty$.

Then $\lim \sup _{n \rightarrow \infty} s_{n}=0$.

Lemma 2.3 (see [12]) In a smooth Banach space $X$, the following inequality holds:

$$
\|x+y\|^{2} \leq\|x\|^{2}+2\langle y, J(x+y)\rangle, \quad \forall x, y \in X
$$

Lemma 2.4 (see [13]) Let $\left\{x_{n}\right\}$ and $\left\{z_{n}\right\}$ be bounded sequences in a Banach space $X$ and let $\left\{\alpha_{n}\right\}$ be a sequence in $[0,1]$ which satisfies the following condition:

$$
0<\liminf _{n \rightarrow \infty} \alpha_{n} \leq \limsup _{n \rightarrow \infty} \alpha_{n}<1 .
$$

Suppose $x_{n+1}=\alpha_{n} x_{n}+\left(1-\alpha_{n}\right) z_{n}, \forall n \geq 0$ and $\lim \sup _{n \rightarrow \infty}\left(\left\|z_{n+1}-z_{n}\right\|-\left\|x_{n+1}-x_{n}\right\|\right) \leq 0$. Then $\lim _{n \rightarrow \infty}\left\|z_{n}-x_{n}\right\|=0$. 
Lemma 2.5 (see [14]) Given a number $r>0$. A real Banach space $X$ is uniformly convex if and only if there exists a continuous strictly increasing function $g:[0, \infty) \rightarrow[0, \infty), g(0)=$ 0 , such that

$$
\|\lambda x+(1-\lambda) y\|^{2} \leq \lambda\|x\|^{2}+(1-\lambda)\|y\|^{2}-\lambda(1-\lambda) g(\|x-y\|)
$$

for all $\lambda \in[0,1]$ and $x, y \in X$ such that $\|x\| \leq r$ and $\|y\| \leq r$.

Lemma 2.6 (see [15]) Let $C$ be a nonempty closed convex subset of a Banach space X. Let $S_{0}, S_{1}, \ldots$ be a sequence of mappings of $C$ into itself. Suppose that $\sum_{n=1}^{\infty} \sup \left\{\left\|S_{n} x-S_{n-1} x\right\|\right.$ : $x \in C\}<\infty$. Then, for each $y \in C,\left\{S_{n} y\right\}$ converges strongly to some point of $C$. Moreover, let $S$ be a mapping of $C$ into itself defined by $S y=\lim _{n \rightarrow \infty} S_{n} y$ for all $y \in C$. Then $\lim _{n \rightarrow \infty} \sup \left\{\left\|S x-S_{n} x\right\|: x \in C\right\}=0$.

Let $C$ be a nonempty closed convex subset of a Banach space $X$ and let $T: C \rightarrow C$ be a nonexpansive mapping with $\operatorname{Fix}(T) \neq \emptyset$. As previously, let $\Xi_{C}$ be a set of all contractions on $C$. For $t \in(0,1)$ and $f \in \Xi_{C}$, let $x_{t} \in C$ be a unique fixed point of the contraction $x \mapsto$ $t f(x)+(1-t) T x$ on $C$; that is,

$$
x_{t}=t f\left(x_{t}\right)+(1-t) T x_{t} .
$$

Lemma 2.7 (see [16]) Let X be a uniformly smooth Banach space, or a reflexive and strictly convex Banach space with a uniformly Gateaux differentiable norm. Let $C$ be a nonempty closed convex subset of $X$, let $T: C \rightarrow C$ be a nonexpansive mapping with $\operatorname{Fix}(T) \neq \emptyset$, and $f \in \Xi_{C}$. Then the net $\left\{x_{t}\right\}$ defined by $x_{t}=t f\left(x_{t}\right)+(1-t) T x_{t}$ converges strongly to a point in $\operatorname{Fix}(T)$. If we define a mapping $Q: \Xi_{C} \rightarrow \operatorname{Fix}(T)$ by $Q(f):=s-\lim _{t \rightarrow 0} x_{t}, \forall f \in \Xi_{C}$, then $Q(f)$ solves the VIP:

$$
\langle(I-f) Q(f), J(Q(f)-p)\rangle \leq 0, \quad \forall f \in \Xi_{C}, p \in \operatorname{Fix}(T) .
$$

Lemma 2.8 (see [17]) Let C be a nonempty closed convex subset of a strictly convex Banach space $X$. Let $\left\{T_{n}\right\}_{n=0}^{\infty}$ be a sequence of nonexpansive mappings on C. Suppose $\bigcap_{n=0}^{\infty} \operatorname{Fix}\left(T_{n}\right)$ is nonempty. Let $\left\{\lambda_{n}\right\}$ be a sequence of positive numbers with $\sum_{n=0}^{\infty} \lambda_{n}=1$. Then a mapping $S$ on $C$ defined by $S x=\sum_{n=0}^{\infty} \lambda_{n} T_{n} x$ for $x \in C$ is well defined, nonexpansive and $\operatorname{Fix}(S)=$ $\bigcap_{n=0}^{\infty} \operatorname{Fix}\left(T_{n}\right)$ holds.

Lemma 2.9 (see [12]) Let $C$ be a nonempty closed convex subset of a smooth Banach space $X$ and let the mapping $B_{i}: C \rightarrow X$ be $\lambda_{i}$-strictly pseudocontractive and $\alpha_{i}$-strongly accretive with $\alpha_{i}+\lambda_{i} \geq 1$ for $i=1,2$. Then, for $\mu_{i} \in(0,1]$, we have

$$
\left\|\left(I-\mu_{i} B_{i}\right) x-\left(I-\mu_{i} B_{i}\right) y\right\| \leq\left\{\sqrt{\frac{1-\alpha_{i}}{\lambda_{i}}}+\left(1-\mu_{i}\right)\left(1+\frac{1}{\lambda_{i}}\right)\right\}\|x-y\|, \quad \forall x, y \in C,
$$

for $i=1$, 2. In particular, if $1-\frac{\lambda_{i}}{1+\lambda_{i}}\left(1-\sqrt{\frac{1-\alpha_{i}}{\lambda_{i}}}\right) \leq \mu_{i} \leq 1$, then $I-\mu_{i} B_{i}$ is nonexpansive for $i=1,2$.

Lemma 2.10 (see [9]) Let $C$ be a nonempty closed convex subset of a smooth Banach space $X$. Let $\Pi_{C}$ be a sunny nonexpansive retraction from $X$ onto $C$ and let the mapping 
$B_{i}: C \rightarrow X$ be $\lambda_{i}$-strictly pseudocontractive and $\alpha_{i}$-strongly accretive with $\alpha_{i}+\lambda_{i} \geq 1$ for $i=1,2$. Let $G: C \rightarrow C$ be a mapping defined by

$$
G(x)=\Pi_{C}\left[\Pi_{C}\left(x-\mu_{2} B_{2} x\right)-\mu_{1} B_{1} \Pi_{C}\left(x-\mu_{2} B_{2} x\right)\right], \quad \forall x \in C .
$$

If $1-\frac{\lambda_{i}}{1+\lambda_{i}}\left(1-\sqrt{\frac{1-\alpha_{i}}{\lambda_{i}}}\right) \leq \mu_{i} \leq 1$, then $G: C \rightarrow C$ is nonexpansive.

Lemma 2.11 (see [9]) Let $C$ be a nonempty closed convex subset of a real 2-uniformly smooth Banach space $X$. Let the mapping $B_{i}: C \rightarrow X$ be $\alpha_{i}$-inverse-strongly accretive. Then we have

$$
\left\|\left(I-\mu_{i} B_{i}\right) x-\left(I-\mu_{i} B_{i}\right) y\right\| \leq\|x-y\|^{2}+2 \mu_{i}\left(\mu_{i} \kappa^{2}-\alpha_{i}\right)\|x-y\|, \quad \forall x, y \in C,
$$

for $i=1,2$, where $\mu_{i}>0$. In particular, if $0<\mu_{i} \leq \frac{\alpha_{i}}{\kappa^{2}}$, then $I-\mu_{i} B_{i}$ is nonexpansive for $i=1,2$.

Lemma 2.12 (see [9]) Let $C$ be a nonempty closed convex subset of a real 2-uniformly smooth Banach space $X$. Let $\Pi_{C}$ be a sunny nonexpansive retraction from $X$ onto $C$. Let the mapping $B_{i}: C \rightarrow X$ be $\alpha_{i}$-inverse-strongly accretive for $i=1$, 2 . Let $\psi: C \rightarrow C$ be the mapping defined by

$$
\psi(x)=\Pi_{C}\left[\Pi_{C}\left(x-\mu_{2} B_{2} x\right)-\mu_{1} B_{1} \Pi_{C}\left(x-\mu_{2} B_{2} x\right)\right], \quad \forall x \in C .
$$

If $0<\mu_{i} \leq \frac{\alpha_{i}}{\kappa^{2}}$ for $i=1,2$, then $\psi: C \rightarrow C$ is nonexpansive.

Lemma 2.13 (see [12]) Let $C$ be a nonempty closed convex subset of a smooth Banach space $X$. Let $\Pi_{C}$ be a sunny nonexpansive retraction from $X$ onto $C$ and let the mapping $B_{i}: C \rightarrow X$ be $\lambda_{i}$-strictly pseudocontractive and $\alpha_{i}$-strongly accretive for $i=1,2$. For given $x^{*}, y^{*} \in C,\left(x^{*}, y^{*}\right)$ is a solution of GSVI (1.1) if and only if $x^{*}=\Pi_{C}\left(y^{*}-\mu_{1} B_{1} y^{*}\right)$, where $y^{*}=\Pi_{C}\left(x^{*}-\mu_{2} B_{2} x^{*}\right)$.

By Lemma 2.12, we observe that

$$
x^{*}=\Pi_{C}\left[\Pi_{C}\left(x^{*}-\mu_{2} B_{2} x^{*}\right)-\mu_{1} B_{1} \Pi_{C}\left(x^{*}-\mu_{2} B_{2} x^{*}\right)\right],
$$

which implies that $x^{*}$ is a fixed point of the mapping $G=\Pi_{C}\left(I-\mu_{1} B_{1}\right) \Pi_{C}\left(I-\mu_{2} B_{2}\right)$.

Proposition 2.1 (see [18]) Let $X$ be a real smooth and uniform convex Banach space and let $r>0$. Then there exists a strictly increasing, continuous and convex function $g:[0,2 r] \rightarrow$ $\mathbf{R}, g(0)=0$ such that

$$
g(\|x-y\|) \leq\|x\|^{2}-2\langle x, J(y)\rangle+\|y\|^{2}, \quad \forall x, y \in B_{r},
$$

where $B_{r}=\{x \in X:\|x\| \leq r\}$. 


\section{Two-step relaxed extragradient algorithms}

In this section, we first suggest and analyze an implicit iterative algorithm by the two-step relaxed extragradient method in the setting of uniformly convex and 2-uniformly smooth Banach spaces, and then another explicit iterative algorithm in the setting of uniformly convex Banach spaces with a uniformly Gateaux differentiable norm.

Theorem 3.1 Let $C$ be a nonempty closed convex subset of a uniformly convex and 2-uniformly smooth Banach space $X$. Let $\Pi_{C}$ be a sunny nonexpansive retraction from $X$ onto $C$. Let the mapping $B_{i}: C \rightarrow X$ be $\alpha_{i}$-inverse-strongly accretive for $i=1,2$. Let $f: C \rightarrow C$ be a contraction with coefficient $\rho \in(0,1)$. Let $\left\{S_{n}\right\}_{n=0}^{\infty}$ be an infinite family of nonexpansive mappings of $C$ into itself such that $F=\bigcap_{i=0}^{\infty} \operatorname{Fix}\left(S_{i}\right) \cap \Omega \neq \emptyset$, where $\Omega$ is a fixed point set of the mapping G. For arbitrarily given $x_{0} \in C$, let $\left\{x_{n}\right\}$ be a sequence generated by

$$
\left\{\begin{array}{l}
y_{n}=\alpha_{n} f\left(y_{n}\right)+\left(1-\alpha_{n}\right) \Pi_{C}\left(I-\mu_{1} B_{1}\right) \Pi_{C}\left(I-\mu_{2} B_{2}\right) x_{n} \\
x_{n+1}=\beta_{n} x_{n}+\left(1-\beta_{n}\right) S_{n} y_{n}, \quad \forall n \geq 0
\end{array}\right.
$$

where $0<\mu_{i}<\frac{\alpha_{i}}{\kappa^{2}}$ for $i=1,2$. Suppose that $\left\{\alpha_{n}\right\}$ and $\left\{\beta_{n}\right\}$ are sequences in $(0,1)$ satisfying the following conditions:

(i) $\lim _{n \rightarrow \infty} \alpha_{n}=0$ and $\sum_{n=0}^{\infty} \alpha_{n}=\infty$;

(ii) $0<\liminf _{n \rightarrow \infty} \beta_{n} \leq \limsup _{n \rightarrow \infty} \beta_{n}<1$.

Assume that $\sum_{n=1}^{\infty} \sup _{x \in D}\left\|S_{n} x-S_{n-1} x\right\|<\infty$ for any bounded subset $D$ of $C$ and let $S$ be a mapping of $C$ into itself defined by $S x=\lim _{n \rightarrow \infty} S_{n} x$ for all $x \in C$ and suppose that $\operatorname{Fix}(S)=$ $\bigcap_{i=0}^{\infty} \operatorname{Fix}\left(S_{i}\right)$. Then $\left\{x_{n}\right\}$ converges strongly to $q \in F$, which solves the following VIP:

$$
\langle q-f(q), J(q-p)\rangle \leq 0, \quad \forall p \in F
$$

Proof It is easy to see that scheme (3.1) can be rewritten as

$$
\left\{\begin{array}{l}
y_{n}=\alpha_{n} f\left(y_{n}\right)+\left(1-\alpha_{n}\right) G\left(x_{n}\right), \\
x_{n+1}=\beta_{n} x_{n}+\left(1-\beta_{n}\right) S_{n} y_{n}, \quad \forall n \geq 0 .
\end{array}\right.
$$

Take a fixed $p \in F$ arbitrarily. Then by Lemma 2.13 we know that $p=G(p)$. Moreover, by Lemma 2.12 we have

$$
\begin{aligned}
\left\|y_{n}-p\right\| & =\left\|\alpha_{n}\left(f\left(y_{n}\right)-p\right)+\left(1-\alpha_{n}\right)\left(G\left(x_{n}\right)-p\right)\right\| \\
& \leq \alpha_{n}\left\|f\left(y_{n}\right)-f(p)\right\|+\alpha_{n}\|f(p)-p\|+\left(1-\alpha_{n}\right)\left\|G\left(x_{n}\right)-p\right\| \\
& \leq \alpha_{n} \rho\left\|y_{n}-p\right\|+\alpha_{n}\|f(p)-p\|+\left(1-\alpha_{n}\right)\left\|x_{n}-p\right\|,
\end{aligned}
$$

which hence implies that

$$
\left\|y_{n}-p\right\| \leq\left(1-\frac{1-\rho}{1-\alpha_{n} \rho} \alpha_{n}\right)\left\|x_{n}-p\right\|+\frac{1}{1-\alpha_{n} \rho} \alpha_{n}\|f(p)-p\| .
$$


Thus, from (3.2) we have

$$
\begin{aligned}
\left\|x_{n+1}-p\right\| \\
\quad=\left\|\beta_{n}\left(x_{n}-p\right)+\left(1-\beta_{n}\right)\left(S_{n} y_{n}-p\right)\right\| \\
\leq \beta_{n}\left\|x_{n}-p\right\|+\left(1-\beta_{n}\right)\left\|S_{n} y_{n}-p\right\| \\
\leq \beta_{n}\left\|x_{n}-p\right\|+\left(1-\beta_{n}\right)\left\|y_{n}-p\right\| \\
\leq \beta_{n}\left\|x_{n}-p\right\|+\left(1-\beta_{n}\right)\left\{\left(1-\frac{1-\rho}{1-\alpha_{n} \rho} \alpha_{n}\right)\left\|x_{n}-p\right\|+\frac{1}{1-\alpha_{n} \rho} \alpha_{n}\|f(p)-p\|\right\} \\
\quad=\left[1-\frac{\left(1-\beta_{n}\right)(1-\rho)}{1-\alpha_{n} \rho} \alpha_{n}\right]\left\|x_{n}-p\right\|+\frac{\left(1-\beta_{n}\right)(1-\rho)}{1-\alpha_{n} \rho} \alpha_{n} \frac{\|f(p)-p\|}{1-\rho} \\
\quad \leq \max \left\{\left\|x_{0}-p\right\|, \frac{\|f(p)-p\|}{1-\rho}\right\} .
\end{aligned}
$$

It immediately follows that $\left\{x_{n}\right\}$ is bounded, and so are the sequences $\left\{y_{n}\right\},\left\{G\left(x_{n}\right)\right\}$ due to (3.3) and the nonexpansivity of $G$.

Let us show that $\left\|x_{n+1}-x_{n}\right\| \rightarrow 0$ as $n \rightarrow \infty$. As a matter of fact, from (3.2) we have

$$
\left\{\begin{array}{l}
y_{n}=\alpha_{n} f\left(y_{n}\right)+\left(1-\alpha_{n}\right) G\left(x_{n}\right) \\
y_{n-1}=\alpha_{n-1} f\left(y_{n-1}\right)+\left(1-\alpha_{n-1}\right) G\left(x_{n-1}\right), \quad \forall n \geq 1
\end{array}\right.
$$

Simple calculations show that

$$
y_{n}-y_{n-1}=\alpha_{n}\left(f\left(y_{n}\right)-f\left(y_{n-1}\right)\right)+\left(\alpha_{n}-\alpha_{n-1}\right)\left(f\left(y_{n-1}\right)-G\left(x_{n-1}\right)\right)+\left(1-\alpha_{n}\right)\left(G\left(x_{n}\right)-G\left(x_{n-1}\right)\right) .
$$

It follows that

$$
\begin{aligned}
\left\|y_{n}-y_{n-1}\right\| \leq & \alpha_{n}\left\|f\left(y_{n}\right)-f\left(y_{n-1}\right)\right\|+\left|\alpha_{n}-\alpha_{n-1}\right|\left\|f\left(y_{n-1}\right)-G\left(x_{n-1}\right)\right\| \\
& +\left(1-\alpha_{n}\right)\left\|G\left(x_{n}\right)-G\left(x_{n-1}\right)\right\| \\
\leq & \alpha_{n} \rho\left\|y_{n}-y_{n-1}\right\|+\left|\alpha_{n}-\alpha_{n-1}\right|\left\|f\left(y_{n-1}\right)-G\left(x_{n-1}\right)\right\| \\
& +\left(1-\alpha_{n}\right)\left\|x_{n}-x_{n-1}\right\|,
\end{aligned}
$$

which hence yields

$$
\left\|y_{n}-y_{n-1}\right\| \leq\left(1-\frac{1-\rho}{1-\alpha_{n} \rho} \alpha_{n}\right)\left\|x_{n}-x_{n-1}\right\|+\frac{\left|\alpha_{n}-\alpha_{n-1}\right|}{1-\alpha_{n} \rho}\left\|f\left(y_{n-1}\right)-G\left(x_{n-1}\right)\right\| .
$$

Thus we have from (3.4)

$$
\begin{aligned}
& \left\|S_{n} y_{n}-S_{n-1} y_{n-1}\right\| \\
& \quad \leq\left\|S_{n} y_{n}-S_{n} y_{n-1}\right\|+\left\|S_{n} y_{n-1}-S_{n-1} y_{n-1}\right\| \\
& \quad \leq\left\|y_{n}-y_{n-1}\right\|+\left\|S_{n} y_{n-1}-S_{n-1} y_{n-1}\right\| \\
& \quad \leq\left(1-\frac{1-\rho}{1-\alpha_{n} \rho} \alpha_{n}\right)\left\|x_{n}-x_{n-1}\right\|+\frac{\left|\alpha_{n}-\alpha_{n-1}\right|}{1-\alpha_{n} \rho}\left\|f\left(y_{n-1}\right)-G\left(x_{n-1}\right)\right\|
\end{aligned}
$$




$$
\begin{aligned}
& +\left\|S_{n} y_{n-1}-S_{n-1} y_{n-1}\right\| \\
\leq & \left\|x_{n}-x_{n-1}\right\|+\frac{\left|\alpha_{n}-\alpha_{n-1}\right|}{1-\alpha_{n} \rho}\left\|f\left(y_{n-1}\right)-G\left(x_{n-1}\right)\right\|+\left\|S_{n} y_{n-1}-S_{n-1} y_{n-1}\right\|,
\end{aligned}
$$

which implies that

$$
\begin{aligned}
& \left\|S_{n} y_{n}-S_{n-1} y_{n-1}\right\|-\left\|x_{n}-x_{n-1}\right\| \\
& \quad \leq \frac{\left|\alpha_{n}-\alpha_{n-1}\right|}{1-\alpha_{n} \rho}\left\|f\left(y_{n-1}\right)-G\left(x_{n-1}\right)\right\|+\left\|S_{n} y_{n-1}-S_{n-1} y_{n-1}\right\| .
\end{aligned}
$$

From condition (i) and the assumption on $\left\{S_{n}\right\}$, we have

$$
\limsup _{n \rightarrow \infty}\left(\left\|S_{n} y_{n}-S_{n-1} y_{n-1}\right\|-\left\|x_{n}-x_{n-1}\right\|\right) \leq 0 .
$$

It follows from Lemma 2.4 that

$$
\lim _{n \rightarrow \infty}\left\|S_{n} y_{n}-x_{n}\right\|=0
$$

Hence we obtain

$$
\lim _{n \rightarrow \infty}\left\|x_{n+1}-x_{n}\right\|=\lim _{n \rightarrow \infty}\left(1-\beta_{n}\right)\left\|S_{n} y_{n}-x_{n}\right\|=0 .
$$

Next we show that $\left\|x_{n}-G\left(x_{n}\right)\right\| \rightarrow 0$ as $n \rightarrow \infty$.

For simplicity, put $q=\Pi_{C}\left(p-\mu_{2} B_{2} p\right), u_{n}=\Pi_{C}\left(x_{n}-\mu_{2} B_{2} x_{n}\right)$ and $v_{n}=\Pi_{C}\left(u_{n}-\mu_{1} B_{1} u_{n}\right)$.

Then $v_{n}=G\left(x_{n}\right)$. From Lemma 2.11 we have

$$
\begin{aligned}
\left\|u_{n}-q\right\|^{2} & =\left\|\Pi_{C}\left(x_{n}-\mu_{2} B_{2} x_{n}\right)-\Pi_{C}\left(p-\mu_{2} B_{2} p\right)\right\|^{2} \\
& \leq\left\|x_{n}-p-\mu_{2}\left(B_{2} x_{n}-B_{2} p\right)\right\|^{2} \\
& \leq\left\|x_{n}-p\right\|^{2}-2 \mu_{2}\left(\alpha_{2}-\kappa^{2} \mu_{2}\right)\left\|B_{2} x_{n}-B_{2} p\right\|^{2}
\end{aligned}
$$

and

$$
\begin{aligned}
\left\|v_{n}-p\right\|^{2} & =\left\|\Pi_{C}\left(u_{n}-\mu_{1} B_{1} u_{n}\right)-\Pi_{C}\left(q-\mu_{1} B_{1} q\right)\right\|^{2} \\
& \leq\left\|u_{n}-q-\mu_{1}\left(B_{1} u_{n}-B_{1} q\right)\right\|^{2} \\
& \leq\left\|u_{n}-q\right\|^{2}-2 \mu_{1}\left(\alpha_{1}-\kappa^{2} \mu_{1}\right)\left\|B_{1} u_{n}-B_{1} q\right\|^{2} .
\end{aligned}
$$

Substituting (3.7) into (3.8), we obtain

$$
\begin{gathered}
\left\|v_{n}-p\right\|^{2} \leq\left\|x_{n}-p\right\|^{2}-2 \mu_{2}\left(\alpha_{2}-\kappa^{2} \mu_{2}\right)\left\|B_{2} x_{n}-B_{2} p\right\|^{2} \\
-2 \mu_{1}\left(\alpha_{1}-\kappa^{2} \mu_{1}\right)\left\|B_{1} u_{n}-B_{1} q\right\|^{2} .
\end{gathered}
$$

According to Lemma 2.3, we have from (3.2)

$$
\begin{aligned}
\left\|y_{n}-p\right\|^{2} & =\left\|\alpha_{n}\left(f\left(y_{n}\right)-f(p)\right)+\left(1-\alpha_{n}\right)\left(v_{n}-p\right)+\alpha_{n}(f(p)-p)\right\|^{2} \\
& \leq\left\|\alpha_{n}\left(f\left(y_{n}\right)-f(p)\right)+\left(1-\alpha_{n}\right)\left(v_{n}-p\right)\right\|^{2}+2 \alpha_{n}\left\langle f(p)-p, J\left(y_{n}-p\right)\right\rangle
\end{aligned}
$$




$$
\begin{aligned}
& \leq \alpha_{n}\left\|f\left(y_{n}\right)-f(p)\right\|^{2}+\left(1-\alpha_{n}\right)\left\|v_{n}-p\right\|^{2}+2 \alpha_{n}\left\langle f(p)-p, J\left(y_{n}-p\right)\right\rangle \\
& \leq \alpha_{n} \rho\left\|y_{n}-p\right\|^{2}+\left(1-\alpha_{n}\right)\left\|v_{n}-p\right\|^{2}+2 \alpha_{n}\|f(p)-p\|\left\|y_{n}-p\right\|,
\end{aligned}
$$

which hence yields

$$
\left\|y_{n}-p\right\|^{2} \leq\left(1-\frac{1-\rho}{1-\alpha_{n} \rho} \alpha_{n}\right)\left\|v_{n}-p\right\|^{2}+\frac{2 \alpha_{n}}{1-\alpha_{n} \rho}\|f(p)-p\|\left\|y_{n}-p\right\| .
$$

From this together with (3.9) and the convexity of $\|\cdot\|^{2}$ we have

$$
\begin{aligned}
&\left\|x_{n+1}-p\right\|^{2} \\
&=\left\|\beta_{n}\left(x_{n}-p\right)+\left(1-\beta_{n}\right)\left(S_{n} y_{n}-p\right)\right\|^{2} \\
& \leq \beta_{n}\left\|x_{n}-p\right\|^{2}+\left(1-\beta_{n}\right)\left\|S_{n} y_{n}-p\right\|^{2} \\
& \leq \beta_{n}\left\|x_{n}-p\right\|^{2}+\left(1-\beta_{n}\right)\left\|y_{n}-p\right\|^{2} \\
& \leq \beta_{n}\left\|x_{n}-p\right\|^{2}+\left(1-\beta_{n}\right)\left\{\left(1-\frac{1-\rho}{1-\alpha_{n} \rho} \alpha_{n}\right)\left\|v_{n}-p\right\|^{2}+\frac{2 \alpha_{n}}{1-\alpha_{n} \rho}\|f(p)-p\|\left\|y_{n}-p\right\|\right\} \\
& \leq \beta_{n}\left\|x_{n}-p\right\|^{2}+\left(1-\beta_{n}\right)\left(1-\frac{1-\rho}{1-\alpha_{n} \rho} \alpha_{n}\right)\left\|v_{n}-p\right\|^{2}+\alpha_{n} M_{1} \\
& \leq \beta_{n}\left\|x_{n}-p\right\|^{2}+\left(1-\beta_{n}\right)\left(1-\frac{1-\rho}{1-\alpha_{n} \rho} \alpha_{n}\right)\left[\left\|x_{n}-p\right\|^{2}\right. \\
&\left.-2 \mu_{2}\left(\alpha_{2}-\kappa^{2} \mu_{2}\right)\left\|B_{2} x_{n}-B_{2} p\right\|^{2}-2 \mu_{1}\left(\alpha_{1}-\kappa^{2} \mu_{1}\right)\left\|B_{1} u_{n}-B_{1} q\right\|^{2}\right]+\alpha_{n} M_{1} \\
&=\left(1-\frac{\left(1-\beta_{n}\right)(1-\rho)}{1-\alpha_{n} \rho} \alpha_{n}\right)\left\|x_{n}-p\right\|^{2} \\
&-2\left(1-\beta_{n}\right)\left(1-\frac{1-\rho}{1-\alpha_{n} \rho} \alpha_{n}\right)\left[\mu_{2}\left(\alpha_{2}-\kappa^{2} \mu_{2}\right)\left\|B_{2} x_{n}-B_{2} p\right\|^{2}\right. \\
&\left.+\mu_{1}\left(\alpha_{1}-\kappa^{2} \mu_{1}\right)\left\|B_{1} u_{n}-B_{1} q\right\|^{2}\right]+\alpha_{n} M_{1} \\
& \leq\left\|x_{n}-p\right\|^{2}-2\left(1-\beta_{n}\right)\left(1-\frac{1-\rho}{1-\alpha_{n} \rho} \alpha_{n}\right)\left[\mu_{2}\left(\alpha_{2}-\kappa^{2} \mu_{2}\right)\left\|B_{2} x_{n}-B_{2} p\right\|^{2}\right. \\
&\left.+\mu_{1}\left(\alpha_{1}-\kappa^{2} \mu_{1}\right)\left\|B_{1} u_{n}-B_{1} q\right\|^{2}\right]+\alpha_{n} M_{1},
\end{aligned}
$$

where $\sup _{n \geq 0}\left\{\frac{2\left(1-\beta_{n}\right)}{1-\alpha_{n} \rho}\|f(p)-p\|\left\|y_{n}-p\right\|\right\} \leq M_{1}$ for some $M_{1}>0$. So, it follows that

$$
\begin{aligned}
2(1- & \left.\beta_{n}\right)\left(1-\frac{1-\rho}{1-\alpha_{n} \rho} \alpha_{n}\right) \\
& \quad \times\left[\mu_{2}\left(\alpha_{2}-\kappa^{2} \mu_{2}\right)\left\|B_{2} x_{n}-B_{2} p\right\|^{2}+\mu_{1}\left(\alpha_{1}-\kappa^{2} \mu_{1}\right)\left\|B_{1} u_{n}-B_{1} q\right\|^{2}\right] \\
\leq & \left\|x_{n}-p\right\|^{2}-\left\|x_{n+1}-p\right\|^{2}+\alpha_{n} M_{1} \\
\leq & \left(\left\|x_{n}-p\right\|+\left\|x_{n+1}-p\right\|\right)\left\|x_{n}-x_{n+1}\right\|+\alpha_{n} M_{1} .
\end{aligned}
$$

Since $0<\mu_{i}<\frac{\alpha_{i}}{\kappa^{2}}$ for $i=1,2$, from conditions (i), (ii) and (3.6) we obtain

$$
\lim _{n \rightarrow \infty}\left\|B_{2} x_{n}-B_{2} p\right\|=0 \quad \text { and } \quad \lim _{n \rightarrow \infty}\left\|B_{1} u_{n}-B_{1} q\right\|=0 .
$$


Utilizing Proposition 2.1 and Lemma 2.1, we have

$$
\begin{aligned}
\left\|u_{n}-q\right\|^{2} \\
\quad=\left\|\Pi_{C}\left(x_{n}-\mu_{2} B_{2} x_{n}\right)-\Pi_{C}\left(p-\mu_{2} B_{2} p\right)\right\|^{2} \\
\quad \leq\left\langle x_{n}-\mu_{2} B_{2} x_{n}-\left(p-\mu_{2} B_{2} p\right), J\left(u_{n}-q\right)\right\rangle \\
\quad=\left\langle x_{n}-p, J\left(u_{n}-q\right)\right\rangle+\mu_{2}\left\langle B_{2} p-B_{2} x_{n}, J\left(u_{n}-q\right)\right\rangle \\
\quad \leq \frac{1}{2}\left[\left\|x_{n}-p\right\|^{2}+\left\|u_{n}-q\right\|^{2}-g_{1}\left(\left\|x_{n}-u_{n}-(p-q)\right\|\right)\right]+\mu_{2}\left\|B_{2} p-B_{2} x_{n}\right\|\left\|u_{n}-q\right\|,
\end{aligned}
$$

which implies that

$$
\left\|u_{n}-q\right\|^{2} \leq\left\|x_{n}-p\right\|^{2}-g_{1}\left(\left\|x_{n}-u_{n}-(p-q)\right\|\right)+2 \mu_{2}\left\|B_{2} p-B_{2} x_{n}\right\|\left\|u_{n}-q\right\| .
$$

In the same way, we derive

$$
\begin{aligned}
& \left\|v_{n}-p\right\|^{2} \\
& \quad=\left\|\Pi_{C}\left(u_{n}-\mu_{1} B_{1} u_{n}\right)-\Pi_{C}\left(q-\mu_{1} B_{1} q\right)\right\|^{2} \\
& \quad \leq\left\langle u_{n}-\mu_{1} B_{1} u_{n}-\left(q-\mu_{1} B_{1} q\right), J\left(v_{n}-p\right)\right\rangle \\
& \quad=\left\langle u_{n}-q, J\left(v_{n}-p\right)\right\rangle+\mu_{1}\left\langle B_{1} q-B_{1} u_{n}, J\left(v_{n}-p\right)\right\rangle \\
& \quad \leq \frac{1}{2}\left[\left\|u_{n}-q\right\|^{2}+\left\|v_{n}-p\right\|^{2}-g_{2}\left(\left\|u_{n}-v_{n}+(p-q)\right\|\right)\right]+\mu_{1}\left\|B_{1} q-B_{1} u_{n}\right\|\left\|v_{n}-p\right\|,
\end{aligned}
$$

which implies that

$$
\left\|v_{n}-p\right\|^{2} \leq\left\|u_{n}-q\right\|^{2}-g_{2}\left(\left\|u_{n}-v_{n}+(p-q)\right\|\right)+2 \mu_{1}\left\|B_{1} q-B_{1} u_{n}\right\|\left\|v_{n}-p\right\| .
$$

Substituting (3.12) into (3.13), we get

$$
\begin{aligned}
\left\|v_{n}-p\right\|^{2} \leq & \left\|x_{n}-p\right\|^{2}-g_{1}\left(\left\|x_{n}-u_{n}-(p-q)\right\|\right)-g_{2}\left(\left\|u_{n}-v_{n}+(p-q)\right\|\right) \\
& +2 \mu_{2}\left\|B_{2} p-B_{2} x_{n}\right\|\left\|u_{n}-q\right\|+2 \mu_{1}\left\|B_{1} q-B_{1} u_{n}\right\|\left\|v_{n}-p\right\| .
\end{aligned}
$$

From (3.10) and (3.14), we have

$$
\begin{aligned}
& \left\|x_{n+1}-p\right\|^{2} \\
& \leq \alpha_{n} M_{1}+\beta_{n}\left\|x_{n}-p\right\|^{2}+\left(1-\beta_{n}\right)\left(1-\frac{1-\rho}{1-\alpha_{n} \rho} \alpha_{n}\right)\left[\left\|x_{n}-p\right\|^{2}\right. \\
& \quad-g_{1}\left(\left\|x_{n}-u_{n}-(p-q)\right\|\right)-g_{2}\left(\left\|u_{n}-v_{n}+(p-q)\right\|\right) \\
& \left.\quad+2 \mu_{2}\left\|B_{2} p-B_{2} x_{n}\right\|\left\|u_{n}-q\right\|+2 \mu_{1}\left\|B_{1} q-B_{1} u_{n}\right\|\left\|v_{n}-p\right\|\right] \\
& \quad \alpha_{n} M_{1}+\left(1-\frac{\left(1-\beta_{n}\right)(1-\rho)}{1-\alpha_{n} \rho} \alpha_{n}\right)\left\|x_{n}-p\right\|^{2} \\
& \quad-\left(1-\beta_{n}\right)\left(1-\frac{1-\rho}{1-\alpha_{n} \rho} \alpha_{n}\right)\left[g_{1}\left(\left\|x_{n}-u_{n}-(p-q)\right\|\right)+g_{2}\left(\left\|u_{n}-v_{n}+(p-q)\right\|\right)\right]
\end{aligned}
$$




$$
\begin{aligned}
& +2 \mu_{2}\left\|B_{2} p-B_{2} x_{n}\right\|\left\|u_{n}-q\right\|+2 \mu_{1}\left\|B_{1} q-B_{1} u_{n}\right\|\left\|v_{n}-p\right\| \\
\leq & \alpha_{n} M_{1}+\left\|x_{n}-p\right\|^{2}-\left(1-\beta_{n}\right)\left(1-\frac{1-\rho}{1-\alpha_{n} \rho} \alpha_{n}\right)\left[g_{1}\left(\left\|x_{n}-u_{n}-(p-q)\right\|\right)\right. \\
& \left.+g_{2}\left(\left\|u_{n}-v_{n}+(p-q)\right\|\right)\right]+2 \mu_{2}\left\|B_{2} p-B_{2} x_{n}\right\|\left\|u_{n}-q\right\| \\
& +2 \mu_{1}\left\|B_{1} q-B_{1} u_{n}\right\|\left\|v_{n}-p\right\|,
\end{aligned}
$$

which implies that

$$
\begin{aligned}
(1- & \left.\beta_{n}\right)\left(1-\frac{1-\rho}{1-\alpha_{n} \rho} \alpha_{n}\right)\left[g_{1}\left(\left\|x_{n}-u_{n}-(p-q)\right\|\right)+g_{2}\left(\left\|u_{n}-v_{n}+(p-q)\right\|\right)\right] \\
\leq & \alpha_{n} M_{1}+\left\|x_{n}-p\right\|^{2}-\left\|x_{n+1}-p\right\|^{2}+2 \mu_{2}\left\|B_{2} p-B_{2} x_{n}\right\|\left\|u_{n}-q\right\| \\
& +2 \mu_{1}\left\|B_{1} q-B_{1} u_{n}\right\|\left\|v_{n}-p\right\| \\
\leq & \alpha_{n} M_{1}+\left(\left\|x_{n}-p\right\|+\left\|x_{n+1}-p\right\|\right)\left\|x_{n}-x_{n+1}\right\|+2 \mu_{2}\left\|B_{2} p-B_{2} x_{n}\right\|\left\|u_{n}-q\right\| \\
& +2 \mu_{1}\left\|B_{1} q-B_{1} u_{n}\right\|\left\|v_{n}-p\right\| .
\end{aligned}
$$

Utilizing conditions (i), (ii), from (3.6) and (3.11) we have

$$
\lim _{n \rightarrow \infty} g_{1}\left(\left\|x_{n}-u_{n}-(p-q)\right\|\right)=0, \quad \lim _{n \rightarrow \infty} g_{2}\left(\left\|u_{n}-v_{n}+(p-q)\right\|\right)=0
$$

Utilizing the properties of $g_{1}$ and $g_{2}$, we deduce that

$$
\lim _{n \rightarrow \infty}\left\|x_{n}-u_{n}-(p-q)\right\|=0, \quad \lim _{n \rightarrow \infty}\left\|u_{n}-v_{n}+(p-q)\right\|=0 .
$$

From (3.16) we obtain

$$
\left\|x_{n}-v_{n}\right\| \leq\left\|x_{n}-u_{n}-(p-q)\right\|+\left\|u_{n}-v_{n}+(p-q)\right\| \rightarrow 0 \quad \text { as } n \rightarrow \infty \text {. }
$$

That is,

$$
\lim _{n \rightarrow \infty}\left\|x_{n}-G\left(x_{n}\right)\right\|=0
$$

On the other hand, we observe that

$$
y_{n}-G\left(x_{n}\right)=\alpha_{n}\left(f\left(y_{n}\right)-G\left(x_{n}\right)\right) \text {. }
$$

Since $\alpha_{n} \rightarrow 0$ as $n \rightarrow \infty$, we have

$$
\lim _{n \rightarrow \infty}\left\|y_{n}-G\left(x_{n}\right)\right\|=0
$$

We note that

$$
\begin{aligned}
\left\|S_{n} G\left(x_{n}\right)-G\left(x_{n}\right)\right\| & \leq\left\|S_{n} G\left(x_{n}\right)-S_{n} y_{n}\right\|+\left\|S_{n} y_{n}-x_{n}\right\|+\left\|x_{n}-G\left(x_{n}\right)\right\| \\
& \leq\left\|G\left(x_{n}\right)-y_{n}\right\|+\left\|S_{n} y_{n}-x_{n}\right\|+\left\|x_{n}-G\left(x_{n}\right)\right\| .
\end{aligned}
$$


From (3.5), (3.17) and (3.18), we obtain that

$$
\lim _{n \rightarrow \infty}\left\|S_{n} G\left(x_{n}\right)-G\left(x_{n}\right)\right\|=0
$$

By (3.19) and Lemma 2.6, we have

$$
\begin{aligned}
\left\|S G\left(x_{n}\right)-G\left(x_{n}\right)\right\| & \leq\left\|S G\left(x_{n}\right)-S_{n} G\left(x_{n}\right)\right\|+\left\|S_{n} G\left(x_{n}\right)-G\left(x_{n}\right)\right\| \\
& \rightarrow 0 \text { as } n \rightarrow \infty .
\end{aligned}
$$

In terms of (3.17) and (3.20), we have

$$
\begin{aligned}
\left\|x_{n}-S x_{n}\right\| & \leq\left\|x_{n}-G\left(x_{n}\right)\right\|+\left\|G\left(x_{n}\right)-S G\left(x_{n}\right)\right\|+\left\|S G\left(x_{n}\right)-S x_{n}\right\| \\
& \leq 2\left\|x_{n}-G\left(x_{n}\right)\right\|+\left\|G\left(x_{n}\right)-S G\left(x_{n}\right)\right\| \\
& \rightarrow 0 \text { as } n \rightarrow \infty .
\end{aligned}
$$

Define a mapping $W x=(1-\theta) S x+\theta G(x), \theta \in(0,1)$ is a constant. Then by Lemma 2.8 we have that $\operatorname{Fix}(W)=\operatorname{Fix}(S) \cap \operatorname{Fix}(G)=F$. We observe that

$$
\begin{aligned}
\left\|x_{n}-W x_{n}\right\| & =\left\|(1-\theta)\left(x_{n}-S x_{n}\right)+\theta\left(x_{n}-G\left(x_{n}\right)\right)\right\| \\
& \leq(1-\theta)\left\|x_{n}-S x_{n}\right\|+\theta\left\|x_{n}-G\left(x_{n}\right)\right\| .
\end{aligned}
$$

From (3.17) and (3.21), we obtain

$$
\lim _{n \rightarrow \infty}\left\|x_{n}-W x_{n}\right\|=0
$$

Now, we claim that

$$
\limsup _{n \rightarrow \infty}\left\langle f(q)-q, J\left(x_{n}-q\right)\right\rangle \leq 0
$$

where $q=s-\lim _{t \rightarrow 0} x_{t}$ with $x_{t}$ being a fixed point of the contraction

$$
x \mapsto t f(x)+(1-t) W x .
$$

Then $x_{t}$ solves the fixed point equation $x_{t}=t f\left(x_{t}\right)+(1-t) W x_{t}$. Thus we have

$$
\left\|x_{t}-x_{n}\right\|=\left\|(1-t)\left(W x_{t}-x_{n}\right)+t\left(f\left(x_{t}\right)-x_{n}\right)\right\| .
$$

By Lemma 2.3 we conclude that

$$
\begin{aligned}
\| x_{t} & -x_{n} \|^{2} \\
& =\left\|(1-t)\left(W x_{t}-x_{n}\right)+t\left(f\left(x_{t}\right)-x_{n}\right)\right\|^{2} \\
& \leq(1-t)^{2}\left\|W x_{t}-x_{n}\right\|^{2}+2 t\left\langle f\left(x_{t}\right)-x_{n}, J\left(x_{t}-x_{n}\right)\right\rangle \\
& \leq(1-t)^{2}\left(\left\|W x_{t}-W x_{n}\right\|+\left\|W x_{n}-x_{n}\right\|\right)^{2}+2 t\left\langle f\left(x_{t}\right)-x_{n}, J\left(x_{t}-x_{n}\right)\right\rangle
\end{aligned}
$$




$$
\begin{aligned}
\leq & (1-t)^{2}\left(\left\|x_{t}-x_{n}\right\|+\left\|W x_{n}-x_{n}\right\|\right)^{2}+2 t\left\langle f\left(x_{t}\right)-x_{n}, J\left(x_{t}-x_{n}\right)\right\rangle \\
= & (1-t)^{2}\left[\left\|x_{t}-x_{n}\right\|^{2}+2\left\|x_{t}-x_{n}\right\|\left\|W x_{n}-x_{n}\right\|+\left\|W x_{n}-x_{n}\right\|^{2}\right] \\
& +2 t\left\langle f\left(x_{t}\right)-x_{t}, J\left(x_{t}-x_{n}\right)\right\rangle+2 t\left\langle x_{t}-x_{n}, J\left(x_{t}-x_{n}\right)\right\rangle \\
& \left(1-2 t+t^{2}\right)\left\|x_{t}-x_{n}\right\|^{2}+f_{n}(t)+2 t\left\langle f\left(x_{t}\right)-x_{t}, J\left(x_{t}-x_{n}\right)\right\rangle+2 t\left\|x_{t}-x_{n}\right\|^{2},
\end{aligned}
$$

where

$$
f_{n}(t)=(1-t)^{2}\left(2\left\|x_{t}-x_{n}\right\|+\left\|x_{n}-W x_{n}\right\|\right)\left\|x_{n}-W x_{n}\right\| \rightarrow 0 \quad \text { as } n \rightarrow \infty \text {. }
$$

It follows from (3.24) that

$$
\left\langle x_{t}-f\left(x_{t}\right), J\left(x_{t}-x_{n}\right)\right\rangle \leq \frac{t}{2}\left\|x_{t}-x_{n}\right\|^{2}+\frac{1}{2 t} f_{n}(t) .
$$

Letting $n \rightarrow \infty$ in (3.26) and noticing (3.25), we derive

$$
\limsup _{n \rightarrow \infty}\left\langle x_{t}-f\left(x_{t}\right), J\left(x_{t}-x_{n}\right)\right\rangle \leq \frac{t}{2} M_{2},
$$

where $M_{2}>0$ is a constant such that $\left\|x_{t}-x_{n}\right\|^{2} \leq M_{2}$ for all $t \in(0,1)$ and $n \geq 0$. Taking $t \rightarrow 0$ in (3.27), we have

$$
\limsup _{t \rightarrow 0} \limsup _{n \rightarrow \infty}\left\langle x_{t}-f\left(x_{t}\right), J\left(x_{t}-x_{n}\right)\right\rangle \leq 0
$$

On the other hand, we have

$$
\begin{aligned}
\langle f(q) & \left.-q, J\left(x_{n}-q\right)\right\rangle \\
= & \left\langle f(q)-q, J\left(x_{n}-q\right)\right\rangle-\left\langle f(q)-q, J\left(x_{n}-x_{t}\right)\right\rangle+\left\langle f(q)-q, J\left(x_{n}-x_{t}\right)\right\rangle \\
& \quad-\left\langle f(q)-x_{t}, J\left(x_{n}-x_{t}\right)\right\rangle+\left\langle f(q)-x_{t}, J\left(x_{n}-x_{t}\right)\right\rangle-\left\langle f\left(x_{t}\right)-x_{t}, J\left(x_{n}-x_{t}\right)\right\rangle \\
& +\left\langle f\left(x_{t}\right)-x_{t}, J\left(x_{n}-x_{t}\right)\right\rangle \\
= & \left\langle f(q)-q, J\left(x_{n}-q\right)-J\left(x_{n}-x_{t}\right)\right\rangle+\left\langle x_{t}-q, J\left(x_{n}-x_{t}\right)\right\rangle \\
& +\left\langle f(q)-f\left(x_{t}\right), J\left(x_{n}-x_{t}\right)\right\rangle+\left\langle f\left(x_{t}\right)-x_{t}, J\left(x_{n}-x_{t}\right)\right\rangle .
\end{aligned}
$$

It follows that

$$
\begin{aligned}
\limsup _{n \rightarrow \infty}\left\langle f(q)-q, J\left(x_{n}-q\right)\right\rangle \leq & \limsup _{n \rightarrow \infty}\left\langle f(q)-q, J\left(x_{n}-q\right)-J\left(x_{n}-x_{t}\right)\right\rangle \\
& +\left\|x_{t}-q\right\| \limsup _{n \rightarrow \infty}\left\|x_{n}-x_{t}\right\|+\rho\left\|q-x_{t}\right\| \limsup _{n \rightarrow \infty}\left\|x_{n}-x_{t}\right\| \\
& +\limsup _{n \rightarrow \infty}\left\langle f\left(x_{t}\right)-x_{t}, J\left(x_{n}-x_{t}\right)\right\rangle .
\end{aligned}
$$

Taking into account that $x_{t} \rightarrow q$ as $t \rightarrow 0$, we have

$$
\begin{aligned}
\limsup _{n \rightarrow \infty}\left\langle f(q)-q, J\left(x_{n}-q\right)\right\rangle & =\underset{t \rightarrow 0}{\limsup } \limsup _{n \rightarrow \infty}\left\langle f(q)-q, J\left(x_{n}-q\right)\right\rangle \\
& \leq \limsup _{t \rightarrow 0} \limsup _{n \rightarrow \infty}\left\langle f(q)-q, J\left(x_{n}-q\right)-J\left(x_{n}-x_{t}\right)\right\rangle .
\end{aligned}
$$


Since $X$ has a uniformly Fréchet differentiable norm, the duality mapping $J$ is norm-tonorm uniformly continuous on bounded subsets of $X$. Consequently, the two limits are interchangeable and hence (3.23) holds. From (3.17) and (3.18) we get $\left(y_{n}-q\right)-\left(x_{n}-q\right) \rightarrow 0$. Noticing that $J$ is norm-to-norm uniformly continuous on bounded subsets of $X$, we deduce from (3.23) that

$$
\begin{aligned}
& \limsup _{n \rightarrow \infty}\left\langle f(q)-q, J\left(y_{n}-q\right)\right\rangle \\
& \quad=\limsup _{n \rightarrow \infty}\left(\left\langle f(q)-q, J\left(x_{n}-q\right)\right\rangle+\left\langle f(q)-q, J\left(y_{n}-q\right)-J\left(x_{n}-q\right)\right\rangle\right) \\
& \quad=\limsup _{n \rightarrow \infty}\left\langle f(q)-q, J\left(x_{n}-q\right)\right\rangle \leq 0 .
\end{aligned}
$$

Finally, let us show that $x_{n} \rightarrow q$ as $n \rightarrow \infty$. We observe that

$$
\begin{aligned}
& \left\|y_{n}-q\right\|^{2} \\
& \quad=\left\|\alpha_{n}\left(f\left(y_{n}\right)-f(q)\right)+\left(1-\alpha_{n}\right)\left(G\left(x_{n}\right)-q\right)+\alpha_{n}(f(q)-q)\right\|^{2} \\
& \quad \leq\left\|\alpha_{n}\left(f\left(y_{n}\right)-f(q)\right)+\left(1-\alpha_{n}\right)\left(G\left(x_{n}\right)-q\right)\right\|^{2}+2 \alpha_{n}\left\langle f(q)-q, J\left(y_{n}-q\right)\right\rangle \\
& \quad \leq \alpha_{n}\left\|f\left(y_{n}\right)-f(q)\right\|^{2}+\left(1-\alpha_{n}\right)\left\|G\left(x_{n}\right)-q\right\|^{2}+2 \alpha_{n}\left\langle f(q)-q, J\left(y_{n}-q\right)\right\rangle \\
& \quad \leq \alpha_{n} \rho\left\|y_{n}-q\right\|^{2}+\left(1-\alpha_{n}\right)\left\|x_{n}-q\right\|^{2}+2 \alpha_{n}\left\langle f(q)-q, J\left(y_{n}-q\right)\right\rangle,
\end{aligned}
$$

which implies that

$$
\left\|y_{n}-q\right\|^{2} \leq\left(1-\frac{1-\rho}{1-\alpha_{n} \rho} \alpha_{n}\right)\left\|x_{n}-q\right\|^{2}+\frac{\alpha_{n}(1-\rho)}{1-\alpha_{n} \rho} \cdot \frac{2\left\langle f(q)-q, J\left(y_{n}-q\right)\right\rangle}{1-\rho}
$$

By the convexity of $\|\cdot\|^{2}$ and (3.2), we get

$$
\left\|x_{n+1}-q\right\|^{2} \leq \beta_{n}\left\|x_{n}-q\right\|^{2}+\left(1-\beta_{n}\right)\left\|y_{n}-q\right\|^{2},
$$

which together with (3.30) leads to

$$
\begin{aligned}
\left\|x_{n+1}-q\right\|^{2} \leq & \beta_{n}\left\|x_{n}-q\right\|^{2}+\left(1-\beta_{n}\right)\left\{\left(1-\frac{1-\rho}{1-\alpha_{n} \rho} \alpha_{n}\right)\left\|x_{n}-q\right\|^{2}\right. \\
& \left.+\frac{\alpha_{n}(1-\rho)}{1-\alpha_{n} \rho} \cdot \frac{2\left\langle f(q)-q, J\left(y_{n}-q\right)\right\rangle}{1-\rho}\right\} \\
= & {\left[1-\frac{\left(1-\beta_{n}\right)(1-\rho)}{1-\alpha_{n} \rho} \alpha_{n}\right]\left\|x_{n}-q\right\|^{2} } \\
& +\frac{\left(1-\beta_{n}\right)(1-\rho)}{1-\alpha_{n} \rho} \alpha_{n} \cdot \frac{2\left\langle f(q)-q, J\left(y_{n}-q\right)\right\rangle}{1-\rho} .
\end{aligned}
$$

Applying Lemma 2.2 to (3.31), we obtain that $x_{n} \rightarrow q$ as $n \rightarrow \infty$. This completes the proof.

Corollary 3.1 Let $C$ be a nonempty closed convex subset of a uniformly convex and 2uniformly smooth Banach space $X$. Let $\Pi_{C}$ be a sunny nonexpansive retraction from $X$ 
onto $C$. Let the mapping $B_{i}: C \rightarrow X$ be $\alpha_{i}$-inverse-strongly accretive for $i=1,2$. Let $f: C \rightarrow$ $C$ be a contraction with coefficient $\rho \in(0,1)$. Let $S$ be a nonexpansive mapping of $C$ into itself such that $F=\operatorname{Fix}(S) \cap \Omega \neq \emptyset$, where $\Omega$ is the fixed point set of the mapping G. For arbitrarily given $x_{0} \in C$, let $\left\{x_{n}\right\}$ be a sequence generated by

$$
\left\{\begin{array}{l}
y_{n}=\alpha_{n} f\left(y_{n}\right)+\left(1-\alpha_{n}\right) \Pi_{C}\left(I-\mu_{1} B_{1}\right) \Pi_{C}\left(I-\mu_{2} B_{2}\right) x_{n} \\
x_{n+1}=\beta_{n} x_{n}+\left(1-\beta_{n}\right) S y_{n}, \quad \forall n \geq 0
\end{array}\right.
$$

where $0<\mu_{i}<\frac{\alpha_{i}}{\kappa^{2}}$ for $i=1,2$. Suppose that $\left\{\alpha_{n}\right\}$ and $\left\{\beta_{n}\right\}$ are sequences in $(0,1)$ satisfying the following conditions:

(i) $\lim _{n \rightarrow \infty} \alpha_{n}=0$ and $\sum_{n=0}^{\infty} \alpha_{n}=\infty$;

(ii) $0<\liminf _{n \rightarrow \infty} \beta_{n} \leq \lim \sup _{n \rightarrow \infty} \beta_{n}<1$.

Then $\left\{x_{n}\right\}$ converges strongly to $q \in F$, which solves the following VIP:

$$
\langle q-f(q), J(q-p)\rangle \leq 0, \quad \forall p \in F
$$

Theorem 3.2 Let $C$ be a nonempty closed convex subset of a uniformly convex Banach space $X$ which has a uniformly Gâteaux differentiable norm. Let $\Pi_{C}$ be a sunny nonexpansive retraction from $X$ onto $C$. Let the mapping $B_{i}: C \rightarrow X$ be $\lambda_{i}$-strictly pseudocontractive and $\alpha_{i}$-strongly accretive with $\alpha_{i}+\lambda_{i} \geq 1$ for $i=1,2$. Let $f: C \rightarrow C$ be a contraction with coefficient $\rho \in(0,1)$. Let $\left\{S_{n}\right\}_{n=0}^{\infty}$ be an infinite family of nonexpansive mappings of $C$ into itself such that $F=\bigcap_{i=0}^{\infty} \operatorname{Fix}\left(S_{i}\right) \cap \Omega \neq \emptyset$, where $\Omega$ is a fixed point set of the mapping G. For arbitrarily given $x_{0} \in C$, let $\left\{x_{n}\right\}$ be a sequence generated by

$$
\left\{\begin{array}{l}
y_{n}=\alpha_{n} x_{n}+\left(1-\alpha_{n}\right) \Pi_{C}\left(I-\mu_{1} B_{1}\right) \Pi_{C}\left(I-\mu_{2} B_{2}\right) x_{n}, \\
x_{n+1}=\beta_{n} f\left(x_{n}\right)+\left(1-\beta_{n}\right) S_{n} y_{n}, \quad \forall n \geq 0,
\end{array}\right.
$$

where $1-\frac{\lambda_{i}}{1+\lambda_{i}}\left(1-\sqrt{\frac{1-\alpha_{i}}{\lambda_{i}}}\right) \leq \mu_{i} \leq 1$ for $i=1,2$. Suppose that $\left\{\alpha_{n}\right\}$ and $\left\{\beta_{n}\right\}$ are sequences in $(0,1)$ satisfying the following conditions:

(i) $0<\liminf _{n \rightarrow \infty} \alpha_{n} \leq \limsup _{n \rightarrow \infty} \alpha_{n}<1$;

(ii) $\lim _{n \rightarrow \infty} \beta_{n}=0$ and $\sum_{n=0}^{\infty} \beta_{n}=\infty$;

(iii) $\sum_{n=1}^{\infty}\left|\alpha_{n}-\alpha_{n-1}\right|<\infty$ or $\lim _{n \rightarrow \infty}\left|\alpha_{n}-\alpha_{n-1}\right| / \beta_{n}=0$;

(iv) $\sum_{n=1}^{\infty}\left|\beta_{n}-\beta_{n-1}\right|<\infty$ or $\lim _{n \rightarrow \infty} \beta_{n-1} / \beta_{n}=1$.

Assume that $\sum_{n=1}^{\infty} \sup _{x \in D}\left\|S_{n} x-S_{n-1} x\right\|<\infty$ for any bounded subset $D$ of $C$ and let $S$ be a mapping of $C$ into itself defined by $S x=\lim _{n \rightarrow \infty} S_{n} x$ for all $x \in C$ and suppose that $\operatorname{Fix}(S)=$ $\bigcap_{i=0}^{\infty} \operatorname{Fix}\left(S_{i}\right)$. Then $\left\{x_{n}\right\}$ converges strongly to $q \in F$, which solves the following VIP:

$$
\langle q-f(q), J(q-p)\rangle \leq 0, \quad \forall p \in F
$$

Proof It is easy to see that scheme (3.32) can be rewritten as

$$
\left\{\begin{array}{l}
y_{n}=\alpha_{n} x_{n}+\left(1-\alpha_{n}\right) G\left(x_{n}\right), \\
x_{n+1}=\beta_{n} f\left(x_{n}\right)+\left(1-\beta_{n}\right) S_{n} y_{n}, \quad \forall n \geq 0 .
\end{array}\right.
$$


Take a fixed $p \in F$ arbitrarily. Then by Lemma 2.13 we know that $p=G(p)$. Moreover, by Lemma 2.10 we have

$$
\begin{aligned}
\left\|y_{n}-p\right\| & =\left\|\alpha_{n}\left(x_{n}-p\right)+\left(1-\alpha_{n}\right)\left(G\left(x_{n}\right)-p\right)\right\| \\
& \leq \alpha_{n}\left\|x_{n}-p\right\|+\left(1-\alpha_{n}\right)\left\|G\left(x_{n}\right)-p\right\| \\
& \leq \alpha_{n}\left\|x_{n}-p\right\|+\left(1-\alpha_{n}\right)\left\|x_{n}-p\right\| \\
& =\left\|x_{n}-p\right\| .
\end{aligned}
$$

From (3.34) we obtain

$$
\begin{aligned}
\left\|x_{n+1}-p\right\| & =\left\|\beta_{n}\left(f\left(x_{n}\right)-p\right)+\left(1-\beta_{n}\right)\left(S_{n} y_{n}-p\right)\right\| \\
& \leq \beta_{n}\left(\left\|f\left(x_{n}\right)-f(p)\right\|+\|f(p)-p\|\right)+\left(1-\beta_{n}\right)\left\|S_{n} y_{n}-p\right\| \\
& \leq \beta_{n} \rho\left\|x_{n}-p\right\|+\beta_{n}\|f(p)-p\|+\left(1-\beta_{n}\right)\left\|y_{n}-p\right\| \\
& \leq \beta_{n} \rho\left\|x_{n}-p\right\|+\beta_{n}\|f(p)-p\|+\left(1-\beta_{n}\right)\left\|x_{n}-p\right\| \\
& =\left(1-\beta_{n}(1-\rho)\right)\left\|x_{n}-p\right\|+\beta_{n}\|f(p)-p\| \\
& =\left(1-\beta_{n}(1-\rho)\right)\left\|x_{n}-p\right\|+\beta_{n}(1-\rho) \cdot \frac{\|f(p)-p\|}{1-\rho} \\
& \leq \max \left\{\left\|x_{0}-p\right\|, \frac{\|f(p)-p\|}{1-\rho}\right\},
\end{aligned}
$$

which implies that $\left\{x_{n}\right\}$ is bounded. By Lemma 2.10 we know from (3.34) that $\left\{G\left(x_{n}\right)\right\}$ and $\left\{y_{n}\right\}$ both are bounded.

Let us show that $\left\|x_{n+1}-x_{n}\right\| \rightarrow 0$ and $\left\|x_{n}-y_{n}\right\| \rightarrow 0$ as $n \rightarrow \infty$. As a matter of fact, from (3.3) we have

$$
\left\{\begin{array}{l}
y_{n}=\alpha_{n} x_{n}+\left(1-\alpha_{n}\right) G\left(x_{n}\right), \\
y_{n-1}=\alpha_{n-1} x_{n-1}+\left(1-\alpha_{n-1}\right) G\left(x_{n-1}\right), \quad \forall n \geq 1 .
\end{array}\right.
$$

Simple calculations show that

$$
y_{n}-y_{n-1}=\alpha_{n}\left(x_{n}-x_{n-1}\right)+\left(\alpha_{n}-\alpha_{n-1}\right)\left(x_{n-1}-G\left(x_{n-1}\right)\right)+\left(1-\alpha_{n}\right)\left(G\left(x_{n}\right)-G\left(x_{n-1}\right)\right) .
$$

It follows that

$$
\begin{aligned}
\left\|y_{n}-y_{n-1}\right\| \leq & \alpha_{n}\left\|x_{n}-x_{n-1}\right\|+\left|\alpha_{n}-\alpha_{n-1}\right|\left\|x_{n-1}-G\left(x_{n-1}\right)\right\| \\
& +\left(1-\alpha_{n}\right)\left\|G\left(x_{n}\right)-G\left(x_{n-1}\right)\right\| \\
\leq & \alpha_{n}\left\|x_{n}-x_{n-1}\right\|+\left|\alpha_{n}-\alpha_{n-1}\right|\left\|x_{n-1}-G\left(x_{n-1}\right)\right\| \\
& +\left(1-\alpha_{n}\right)\left\|x_{n}-x_{n-1}\right\| \\
= & \left\|x_{n}-x_{n-1}\right\|+\left|\alpha_{n}-\alpha_{n-1}\right|\left\|x_{n-1}-G\left(x_{n-1}\right)\right\| .
\end{aligned}
$$


Furthermore, from (3.33) we have

$$
\left\{\begin{array}{l}
x_{n+1}=\beta_{n} f\left(x_{n}\right)+\left(1-\beta_{n}\right) S_{n} y_{n}, \\
x_{n}=\beta_{n-1} f\left(x_{n-1}\right)+\left(1-\beta_{n-1}\right) S_{n-1} y_{n-1}, \quad \forall n \geq 1 .
\end{array}\right.
$$

Also, simple calculations show that

$$
\begin{aligned}
x_{n+1}-x_{n}= & \beta_{n}\left(f\left(x_{n}\right)-f\left(x_{n-1}\right)\right)+\left(\beta_{n}-\beta_{n-1}\right)\left(f\left(x_{n-1}\right)-S_{n-1} y_{n-1}\right) \\
& +\left(1-\beta_{n}\right)\left(S_{n} y_{n}-S_{n-1} y_{n-1}\right) .
\end{aligned}
$$

It follows from (3.35) and (3.36) that

$$
\begin{aligned}
\left\|x_{n+1}-x_{n}\right\| \leq & \beta_{n}\left\|f\left(x_{n}\right)-f\left(x_{n-1}\right)\right\|+\left|\beta_{n}-\beta_{n-1}\right|\left\|f\left(x_{n-1}\right)-S_{n-1} y_{n-1}\right\| \\
& +\left(1-\beta_{n}\right)\left\|S_{n} y_{n}-S_{n-1} y_{n-1}\right\| \\
\leq & \beta_{n} \rho\left\|x_{n}-x_{n-1}\right\|+\left|\beta_{n}-\beta_{n-1}\right|\left\|f\left(x_{n-1}\right)-S_{n-1} y_{n-1}\right\| \\
& +\left(1-\beta_{n}\right)\left(\left\|S_{n} y_{n}-S_{n} y_{n-1}\right\|+\left\|S_{n} y_{n-1}-S_{n-1} y_{n-1}\right\|\right) \\
\leq & \beta_{n} \rho\left\|x_{n}-x_{n-1}\right\|+\left|\beta_{n}-\beta_{n-1}\right|\left\|f\left(x_{n-1}\right)-S_{n-1} y_{n-1}\right\| \\
& +\left(1-\beta_{n}\right)\left(\left\|y_{n}-y_{n-1}\right\|+\left\|S_{n} y_{n-1}-S_{n-1} y_{n-1}\right\|\right) \\
\leq & \beta_{n} \rho\left\|x_{n}-x_{n-1}\right\|+\left|\beta_{n}-\beta_{n-1}\right|\left\|f\left(x_{n-1}\right)-S_{n-1} y_{n-1}\right\| \\
& +\left(1-\beta_{n}\right)\left[\left\|x_{n}-x_{n-1}\right\|+\left|\alpha_{n}-\alpha_{n-1}\right|\left\|x_{n-1}-G\left(x_{n-1}\right)\right\|\right] \\
& +\left\|S_{n} y_{n-1}-S_{n-1} y_{n-1}\right\| \\
= & \left(1-\beta_{n}(1-\rho)\right)\left\|x_{n}-x_{n-1}\right\|+\left|\beta_{n}-\beta_{n-1}\right|\left\|f\left(x_{n-1}\right)-S_{n-1} y_{n-1}\right\| \\
& +\left|\alpha_{n}-\alpha_{n-1}\right|\left\|x_{n-1}-G\left(x_{n-1}\right)\right\|+\left\|S_{n} y_{n-1}-S_{n-1} y_{n-1}\right\| \\
\leq & \left(1-\beta_{n}(1-\rho)\right)\left\|x_{n}-x_{n-1}\right\|+M\left(\left|\alpha_{n}-\alpha_{n-1}\right|+\left|\beta_{n}-\beta_{n-1}\right|\right) \\
& +\left\|S_{n} y_{n-1}-S_{n-1} y_{n-1}\right\|,
\end{aligned}
$$

where $\sup _{n \geq 0}\left\{\left\|f\left(x_{n}\right)-S_{n} y_{n}\right\|+\left\|x_{n}-G\left(x_{n}\right)\right\|\right\} \leq M$ for some $M>0$. Utilizing Lemma 2.2, from conditions (ii)-(iv) and the assumption on $\left\{S_{n}\right\}$, we deduce that

$$
\lim _{n \rightarrow \infty}\left\|x_{n+1}-x_{n}\right\|=0
$$

Since $\left\{x_{n}\right\}$ and $\left\{G\left(x_{n}\right)\right\}$ both are bounded, by Lemma 2.5 there exists a continuous strictly increasing function $g:[0, \infty) \rightarrow[0, \infty), g(0)=0$ such that for $p \in F$

$$
\begin{aligned}
& \left\|y_{n}-p\right\|^{2} \\
& \quad \leq \alpha_{n}\left\|x_{n}-p\right\|^{2}+\left(1-\alpha_{n}\right)\left\|G\left(x_{n}\right)-p\right\|^{2}-\alpha_{n}\left(1-\alpha_{n}\right) g\left(\left\|x_{n}-G\left(x_{n}\right)\right\|\right) \\
& \quad \leq \alpha_{n}\left\|x_{n}-p\right\|^{2}+\left(1-\alpha_{n}\right)\left\|x_{n}-p\right\|^{2}-\alpha_{n}\left(1-\alpha_{n}\right) g\left(\left\|x_{n}-G\left(x_{n}\right)\right\|\right) \\
& \quad=\left\|x_{n}-p\right\|^{2}-\alpha_{n}\left(1-\alpha_{n}\right) g\left(\left\|x_{n}-G\left(x_{n}\right)\right\|\right),
\end{aligned}
$$


which together with (3.33) implies that

$$
\begin{aligned}
&\left\|x_{n+1}-p\right\|^{2} \\
&=\left\|\beta_{n}\left(f\left(x_{n}\right)-f(p)\right)+\left(1-\beta_{n}\right)\left(S_{n} y_{n}-p\right)+\beta_{n}(f(p)-p)\right\|^{2} \\
& \leq\left\|\beta_{n}\left(f\left(x_{n}\right)-f(p)\right)+\left(1-\beta_{n}\right)\left(S_{n} y_{n}-p\right)\right\|^{2}+2 \beta_{n}\left\langle f(p)-p, J\left(x_{n+1}-p\right)\right\rangle \\
& \leq \beta_{n}\left\|f\left(x_{n}\right)-f(p)\right\|^{2}+\left(1-\beta_{n}\right)\left\|S_{n} y_{n}-p\right\|^{2}+2 \beta_{n}\|f(p)-p\|\left\|x_{n+1}-p\right\| \\
& \leq \beta_{n} \rho^{2}\left\|x_{n}-p\right\|^{2}+\left(1-\beta_{n}\right)\left\|y_{n}-p\right\|^{2}+2 \beta_{n}\|f(p)-p\|\left\|x_{n+1}-p\right\| \\
& \leq \beta_{n} \rho\left\|x_{n}-p\right\|^{2}+\left(1-\beta_{n}\right)\left[\left\|x_{n}-p\right\|^{2}-\alpha_{n}\left(1-\alpha_{n}\right) g\left(\left\|x_{n}-G\left(x_{n}\right)\right\|\right)\right] \\
&+2 \beta_{n}\|f(p)-p\|\left\|x_{n+1}-p\right\| \\
&=\left(1-\beta_{n}(1-\rho)\right)\left\|x_{n}-p\right\|^{2}-\left(1-\beta_{n}\right) \alpha_{n}\left(1-\alpha_{n}\right) g\left(\left\|x_{n}-G\left(x_{n}\right)\right\|\right) \\
&+2 \beta_{n}\|f(p)-p\|\left\|x_{n+1}-p\right\| \\
& \leq\left\|x_{n}-p\right\|^{2}-\left(1-\beta_{n}\right) \alpha_{n}\left(1-\alpha_{n}\right) g\left(\left\|x_{n}-G\left(x_{n}\right)\right\|\right)+2 \beta_{n}\|f(p)-p\|\left\|x_{n+1}-p\right\| .
\end{aligned}
$$

It immediately follows that

$$
\begin{aligned}
& \left(1-\beta_{n}\right) \alpha_{n}\left(1-\alpha_{n}\right) g\left(\left\|x_{n}-G\left(x_{n}\right)\right\|\right) \\
& \quad \leq\left\|x_{n}-p\right\|^{2}-\left\|x_{n+1}-p\right\|^{2}+2 \beta_{n}\|f(p)-p\|\left\|x_{n+1}-p\right\| \\
& \quad \leq\left(\left\|x_{n}-p\right\|+\left\|x_{n+1}-p\right\|\right)\left\|x_{n}-x_{n+1}\right\|+2 \beta_{n}\|f(p)-p\|\left\|x_{n+1}-p\right\| .
\end{aligned}
$$

Since $\beta_{n} \rightarrow 0,\left\|x_{n+1}-x_{n}\right\| \rightarrow 0$ and $0<\liminf _{n \rightarrow \infty} \alpha_{n} \leq \limsup _{n \rightarrow \infty} \alpha_{n}<1$, we get $\lim _{n \rightarrow \infty} g\left(\left\|x_{n}-G\left(x_{n}\right)\right\|\right)=0$ and hence

$$
\lim _{n \rightarrow \infty}\left\|x_{n}-G\left(x_{n}\right)\right\|=0 .
$$

Thus, from (3.33) and (3.40) it follows that

$$
\lim _{n \rightarrow \infty}\left\|y_{n}-x_{n}\right\|=\lim _{n \rightarrow \infty}\left(1-\alpha_{n}\right)\left\|G\left(x_{n}\right)-x_{n}\right\|=0 .
$$

On the other hand, we observe that

$$
\begin{aligned}
x_{n+1}-x_{n} & =\beta_{n}\left(f\left(x_{n}\right)-x_{n}\right)+\left(1-\beta_{n}\right)\left(S_{n} y_{n}-x_{n}\right) \\
& =\beta_{n}\left(f\left(x_{n}\right)-x_{n}\right)+\left(1-\beta_{n}\right)\left(S_{n} y_{n}-y_{n}\right)+\left(1-\beta_{n}\right)\left(y_{n}-x_{n}\right) .
\end{aligned}
$$

Then we have

$$
\begin{aligned}
& \left(1-\beta_{n}\right)\left\|S_{n} y_{n}-y_{n}\right\| \\
& \quad=\left\|x_{n+1}-x_{n}-\beta_{n}\left(f\left(x_{n}\right)-x_{n}\right)-\left(1-\beta_{n}\right)\left(y_{n}-x_{n}\right)\right\| \\
& \quad \leq\left\|x_{n+1}-x_{n}\right\|+\beta_{n}\left\|f\left(x_{n}\right)-x_{n}\right\|+\left(1-\beta_{n}\right)\left\|y_{n}-x_{n}\right\| \\
& \quad \leq\left\|x_{n+1}-x_{n}\right\|+\beta_{n}\left\|f\left(x_{n}\right)-x_{n}\right\|+\left\|y_{n}-x_{n}\right\| .
\end{aligned}
$$


Since $\beta_{n} \rightarrow 0,\left\|x_{n+1}-x_{n}\right\| \rightarrow 0$ and $\left\|x_{n}-y_{n}\right\| \rightarrow 0$ as $n \rightarrow \infty$, we get

$$
\lim _{n \rightarrow \infty}\left\|S_{n} y_{n}-y_{n}\right\|=0 \quad \text { and } \quad \lim _{n \rightarrow \infty}\left\|S_{n} y_{n}-x_{n}\right\|=0 .
$$

In the meantime, since $\left\|x_{n}-G\left(x_{n}\right)\right\| \rightarrow 0$ and $\left\|x_{n}-y_{n}\right\| \rightarrow 0$ as $n \rightarrow \infty$, we also get

$$
\lim _{n \rightarrow \infty}\left\|y_{n}-G\left(x_{n}\right)\right\|=0 .
$$

We note that

$$
\begin{aligned}
\left\|S_{n} G\left(x_{n}\right)-G\left(x_{n}\right)\right\| & \leq\left\|S_{n} G\left(x_{n}\right)-S_{n} y_{n}\right\|+\left\|S_{n} y_{n}-x_{n}\right\|+\left\|x_{n}-G\left(x_{n}\right)\right\| \\
& \leq\left\|G\left(x_{n}\right)-y_{n}\right\|+\left\|S_{n} y_{n}-x_{n}\right\|+\left\|x_{n}-G\left(x_{n}\right)\right\| .
\end{aligned}
$$

From (3.40), (3.42) and (3.43), we obtain

$$
\lim _{n \rightarrow \infty}\left\|S_{n} G\left(x_{n}\right)-G\left(x_{n}\right)\right\|=0 .
$$

By (3.44) and Lemma 2.6, we have

$$
\begin{aligned}
\left\|S G\left(x_{n}\right)-G\left(x_{n}\right)\right\| & \leq\left\|S G\left(x_{n}\right)-S_{n} G\left(x_{n}\right)\right\|+\left\|S_{n} G\left(x_{n}\right)-G\left(x_{n}\right)\right\| \\
& \rightarrow 0 \quad \text { as } n \rightarrow \infty .
\end{aligned}
$$

In terms of (3.40) and (3.45), we have

$$
\begin{aligned}
\left\|x_{n}-S x_{n}\right\| & \leq\left\|x_{n}-G\left(x_{n}\right)\right\|+\left\|G\left(x_{n}\right)-S G\left(x_{n}\right)\right\|+\left\|S G\left(x_{n}\right)-S x_{n}\right\| \\
& \leq 2\left\|x_{n}-G\left(x_{n}\right)\right\|+\left\|G\left(x_{n}\right)-S G\left(x_{n}\right)\right\| \\
& \rightarrow 0 \quad \text { as } n \rightarrow \infty .
\end{aligned}
$$

Define a mapping $W x=(1-\theta) S x+\theta G(x), \theta \in(0,1)$ is a constant. Then by Lemma 2.8 we have that $\operatorname{Fix}(W)=\operatorname{Fix}(S) \cap \operatorname{Fix}(G)=F$. We observe that

$$
\begin{aligned}
\left\|x_{n}-W x_{n}\right\| & =\left\|(1-\theta)\left(x_{n}-S x_{n}\right)+\theta\left(x_{n}-G\left(x_{n}\right)\right)\right\| \\
& \leq(1-\theta)\left\|x_{n}-S x_{n}\right\|+\theta\left\|x_{n}-G\left(x_{n}\right)\right\| .
\end{aligned}
$$

From (3.40) and (3.46), we obtain

$$
\lim _{n \rightarrow \infty}\left\|x_{n}-W x_{n}\right\|=0
$$

Now, we claim that

$$
\limsup _{n \rightarrow \infty}\left\langle f(q)-q, J\left(x_{n}-q\right)\right\rangle \leq 0
$$

where $q=s-\lim _{t \rightarrow 0} x_{t}$ with $x_{t}$ being the fixed point of the contraction $x \mapsto t f(x)+(1-t) W x$. Then $x_{t}$ solves the fixed point equation $x_{t}=t f\left(x_{t}\right)+(1-t) W x_{t}$. Utilizing the arguments 
similar to those of (3.28) in the proof of Theorem 3.1, we can deduce that

$$
\begin{aligned}
\limsup _{n \rightarrow \infty}\left\langle f(q)-q, J\left(x_{n}-q\right)\right\rangle & =\limsup _{t \rightarrow 0} \limsup _{n \rightarrow \infty}\left\langle f(q)-q, J\left(x_{n}-q\right)\right\rangle \\
& \leq \limsup _{t \rightarrow 0} \limsup _{n \rightarrow \infty}\left\langle f(q)-q, J\left(x_{n}-q\right)-J\left(x_{n}-x_{t}\right)\right\rangle .
\end{aligned}
$$

Since $X$ has a uniformly Gâteaux differentiable norm, the duality mapping $J$ is norm-toweak* uniformly continuous on bounded subsets of $X$. Consequently, the two limits are interchangeable and hence the following holds:

$$
\limsup _{n \rightarrow \infty}\left\langle f(q)-q, J\left(x_{n}-q\right)\right\rangle \leq 0
$$

From (3.38) we get $\left(x_{n+1}-q\right)-\left(x_{n}-q\right) \rightarrow 0$. Noticing the norm-to-weak* uniform continuity of $J$ on bounded subsets of $X$, we deduce from (3.48) that

$$
\begin{aligned}
& \limsup _{n \rightarrow \infty}\left\langle f(q)-q, J\left(x_{n+1}-q\right)\right\rangle \\
& \quad=\limsup _{n \rightarrow \infty}\left(\left\langle f(q)-q, J\left(x_{n+1}-q\right)-J\left(x_{n}-q\right)\right\rangle+\left\langle f(q)-q, J\left(x_{n+1}-q\right)\right\rangle\right) \\
& \quad=\limsup _{n \rightarrow \infty}\left\langle f(q)-q, J\left(x_{n}-q\right)\right\rangle \leq 0 .
\end{aligned}
$$

Finally, let us show that $x_{n} \rightarrow q$ as $n \rightarrow \infty$. We observe that

$$
\begin{aligned}
\left\|y_{n}-q\right\| & =\left\|\alpha_{n}\left(x_{n}-q\right)+\left(1-\alpha_{n}\right)\left(G\left(x_{n}\right)-q\right)\right\| \\
& \leq \alpha_{n}\left\|x_{n}-q\right\|+\left(1-\alpha_{n}\right)\left\|x_{n}-q\right\|=\left\|x_{n}-q\right\|,
\end{aligned}
$$

and

$$
\begin{aligned}
& \left\|x_{n+1}-q\right\|^{2} \\
& \quad=\left\|\beta_{n}\left(f\left(x_{n}\right)-f(q)\right)+\left(1-\beta_{n}\right)\left(S_{n} y_{n}-q\right)+\beta_{n}(f(q)-q)\right\|^{2} \\
& \quad \leq\left\|\beta_{n}\left(f\left(x_{n}\right)-f(q)\right)+\left(1-\beta_{n}\right)\left(S_{n} y_{n}-q\right)\right\|^{2}+2 \beta_{n}\left\langle f(q)-q, J\left(x_{n+1}-q\right)\right\rangle \\
& \quad \leq \beta_{n}\left\|f\left(x_{n}\right)-f(q)\right\|^{2}+\left(1-\beta_{n}\right)\left\|S_{n} y_{n}-q\right\|^{2}+2 \beta_{n}\left\langle f(q)-q, J\left(x_{n+1}-q\right)\right\rangle \\
& \quad \leq \beta_{n} \rho\left\|x_{n}-q\right\|^{2}+\left(1-\beta_{n}\right)\left\|y_{n}-q\right\|^{2}+2 \beta_{n}\left\langle f(q)-q, J\left(x_{n+1}-q\right)\right\rangle \\
& \quad \leq \beta_{n} \rho\left\|x_{n}-q\right\|^{2}+\left(1-\beta_{n}\right)\left\|x_{n}-q\right\|^{2}+2 \beta_{n}\left\langle f(q)-q, J\left(x_{n+1}-q\right)\right\rangle \\
& \quad=\left(1-\beta_{n}(1-\rho)\right)\left\|x_{n}-q\right\|^{2}+2 \beta_{n}\left\langle f(q)-q, J\left(x_{n+1}-q\right)\right\rangle .
\end{aligned}
$$

Since $\sum_{n=0}^{\infty} \beta_{n}=\infty$ and $\lim \sup _{n \rightarrow \infty}\left\langle f(q)-q, J\left(x_{n+1}-q\right)\right\rangle \leq 0$, by Lemma 2.2 we conclude from (3.51) that $x_{n} \rightarrow q$ as $n \rightarrow \infty$. This completes the proof.

Corollary 3.2 Let $C$ be a nonempty closed convex subset of a uniformly convex Banach space $X$ which has a uniformly Gâteaux differentiable norm. Let $\Pi_{C}$ be a sunny nonexpansive retraction from $X$ onto $C$. Let the mapping $B_{i}: C \rightarrow X$ be $\lambda_{i}$-strictly pseudocontractive and $\alpha_{i}$-strongly accretive with $\alpha_{i}+\lambda_{i} \geq 1$ for $i=1,2$. Let $f: C \rightarrow C$ be a contraction with coefficient $\rho \in(0,1)$. Let $S$ be a nonexpansive mapping of $C$ into itself such that 
$F=\operatorname{Fix}(S) \cap \Omega \neq \emptyset$, where $\Omega$ is a fixed point set of the mapping G. For arbitrarily given $x_{0} \in C$, let $\left\{x_{n}\right\}$ be a sequence generated by

$$
\left\{\begin{array}{l}
y_{n}=\alpha_{n} x_{n}+\left(1-\alpha_{n}\right) \Pi_{C}\left(I-\mu_{1} B_{1}\right) \Pi_{C}\left(I-\mu_{2} B_{2}\right) x_{n} \\
x_{n+1}=\beta_{n} f\left(x_{n}\right)+\left(1-\beta_{n}\right) S y_{n}, \quad \forall n \geq 0
\end{array}\right.
$$

where $1-\frac{\lambda_{i}}{1+\lambda_{i}}\left(1-\sqrt{\frac{1-\alpha_{i}}{\lambda_{i}}}\right) \leq \mu_{i} \leq 1$ for $i=1,2$. Suppose that $\left\{\alpha_{n}\right\}$ and $\left\{\beta_{n}\right\}$ are sequences in $(0,1)$ satisfying the following conditions:

(i) $0<\liminf _{n \rightarrow \infty} \alpha_{n} \leq \limsup _{n \rightarrow \infty} \alpha_{n}<1$;

(ii) $\lim _{n \rightarrow \infty} \beta_{n}=0$ and $\sum_{n=0}^{\infty} \beta_{n}=\infty$;

(iii) $\sum_{n=1}^{\infty}\left|\alpha_{n}-\alpha_{n-1}\right|<\infty$ or $\lim _{n \rightarrow \infty}\left|\alpha_{n}-\alpha_{n-1}\right| / \beta_{n}=0$;

(iv) $\sum_{n=1}^{\infty}\left|\beta_{n}-\beta_{n-1}\right|<\infty$ or $\lim _{n \rightarrow \infty} \beta_{n-1} / \beta_{n}=1$.

Then $\left\{x_{n}\right\}$ converges strongly to $q \in F$, which solves the following VIP:

$$
\langle q-f(q), J(q-p)\rangle \leq 0, \quad \forall p \in F
$$

Remark 3.1 Theorems 3.1 and 3.2 improve, extend, supplement and develop [14, Theorem 3.1] in the following aspects. Although the iterative algorithm in Theorem 3.1 is an implicit algorithm, we can derive the strong convergence of the proposed algorithm under the same conditions on the parameter sequences $\left\{\alpha_{n}\right\},\left\{\beta_{n}\right\}$ as in [14, Theorem 3.1]. The assumption of the uniformly convex and 2-uniformly smooth Banach space $X$ in [14, Theorem 3.1] is weakened to the one of the uniformly convex Banach space $X$ having a uniformly Gâteaux differentiable norm in Theorem 3.2.

\section{Relaxed extragradient composite algorithms}

In this section, we propose and analyze a composite explicit iterative algorithm by the two-step relaxed extragradient method for solving GSVI (1.1) and the common fixed point problem of an infinite family of nonexpansive self-mappings $\left\{S_{n}\right\}$ in a 2-uniformly smooth and uniformly convex Banach space.

Theorem 4.1 Let $C$ be a nonempty closed convex subset of a uniformly convex and 2uniformly smooth Banach space $X$. Let $\Pi_{C}$ be a sunny nonexpansive retraction from $X$ onto $C$. Let the mapping $B_{i}: C \rightarrow X$ be $\alpha_{i}$-inverse-strongly accretive for $i=1,2$. Let $f: C \rightarrow$ $C$ be a contraction with coefficient $\rho \in(0,1)$. Let $\left\{S_{n}\right\}_{n=0}^{\infty}$ be an infinite family of nonexpansive mappings of $C$ into itself such that $F=\bigcap_{i=0}^{\infty} \operatorname{Fix}\left(S_{i}\right) \cap \Omega \neq \emptyset$, where $\Omega$ is a fixed point set of the mapping G. For arbitrarily given $x_{0} \in C$, let $\left\{x_{n}\right\}$ be a sequence generated by

$$
\left\{\begin{array}{l}
y_{n}=\alpha_{n} f\left(x_{n}\right)+\left(1-\alpha_{n}\right) S_{n} \Pi_{C}\left(I-\mu_{1} B_{1}\right) \Pi_{C}\left(I-\mu_{2} B_{2}\right) x_{n}, \\
x_{n+1}=\beta_{n} y_{n}+\left(1-\beta_{n}\right) S_{n} \Pi_{C}\left(I-\mu_{1} B_{1}\right) \Pi_{C}\left(I-\mu_{2} B_{2}\right) y_{n}, \quad \forall n \geq 0,
\end{array}\right.
$$

where $0<\mu_{i}<\frac{\alpha_{i}}{\kappa^{2}}$ for $i=1,2$. Suppose that $\left\{\alpha_{n}\right\}$ and $\left\{\beta_{n}\right\}$ are sequences in $(0,1]$ satisfying the following conditions:

(i) $\lim _{n \rightarrow \infty} \alpha_{n}=0$ and $\sum_{n=0}^{\infty} \alpha_{n}=\infty$;

(ii) $\left\{\beta_{n}\right\} \subset[a, 1]$ for some $a \in(0,1)$; 
(iii) $\sum_{n=1}^{\infty}\left|\alpha_{n}-\alpha_{n-1}\right|<\infty$ or $\lim _{n \rightarrow \infty} \alpha_{n-1} / \alpha_{n}=1$;

(iv) $\sum_{n=1}^{\infty}\left|\beta_{n}-\beta_{n-1}\right|<\infty$ or $\lim _{n \rightarrow \infty}\left|\beta_{n}-\beta_{n-1}\right| / \alpha_{n}=0$.

Assume that $\sum_{n=1}^{\infty} \sup _{x \in D}\left\|S_{n} x-S_{n-1} x\right\|<\infty$ for any bounded subset $D$ of $C$ and let $S$ be a mapping of C into itself defined by $S x=\lim _{n \rightarrow \infty} S_{n} x$ for all $x \in C$ and suppose that $\operatorname{Fix}(S)=$ $\bigcap_{i=0}^{\infty} \operatorname{Fix}\left(S_{i}\right)$. Then $\left\{x_{n}\right\}$ converges strongly to $q \in F$, which solves the following VIP:

$$
\langle q-f(q), J(q-p)\rangle \leq 0, \quad \forall p \in F .
$$

Proof It is easy to see that scheme (4.1) can be rewritten as

$$
\left\{\begin{array}{l}
y_{n}=\alpha_{n} f\left(x_{n}\right)+\left(1-\alpha_{n}\right) S_{n} G\left(x_{n}\right), \\
x_{n+1}=\beta_{n} y_{n}+\left(1-\beta_{n}\right) S_{n} G\left(y_{n}\right), \quad \forall n \geq 0 .
\end{array}\right.
$$

Take a fixed $p \in F$ arbitrarily. Then by Lemma 2.13 we know that $p=G(p)$. Moreover, by Lemma 2.12 we have

$$
\begin{aligned}
\left\|y_{n}-p\right\| & =\left\|\alpha_{n}\left(f\left(x_{n}\right)-p\right)+\left(1-\alpha_{n}\right)\left(S_{n} G\left(x_{n}\right)-p\right)\right\| \\
& \leq \alpha_{n}\left\|f\left(x_{n}\right)-f(p)\right\|+\alpha_{n}\|f(p)-p\|+\left(1-\alpha_{n}\right)\left\|S_{n} G\left(x_{n}\right)-p\right\| \\
& \leq \alpha_{n} \rho\left\|x_{n}-p\right\|+\alpha_{n}\|f(p)-p\|+\left(1-\alpha_{n}\right)\left\|G\left(x_{n}\right)-p\right\| \\
& \leq \alpha_{n} \rho\left\|x_{n}-p\right\|+\alpha_{n}\|f(p)-p\|+\left(1-\alpha_{n}\right)\left\|x_{n}-p\right\| \\
& =\left(1-\alpha_{n}(1-\rho)\right)\left\|x_{n}-p\right\|+\alpha_{n}\|f(p)-p\| .
\end{aligned}
$$

From (4.3) we have

$$
\begin{aligned}
\left\|x_{n+1}-p\right\| & =\left\|\beta_{n}\left(y_{n}-p\right)+\left(1-\beta_{n}\right)\left(S_{n} G\left(y_{n}\right)-p\right)\right\| \\
& \leq \beta_{n}\left\|y_{n}-p\right\|+\left(1-\beta_{n}\right)\left\|S_{n} G\left(y_{n}\right)-p\right\| \\
& \leq \beta_{n}\left\|y_{n}-p\right\|+\left(1-\beta_{n}\right)\left\|G\left(y_{n}\right)-p\right\| \\
& \leq \beta_{n}\left\|y_{n}-p\right\|+\left(1-\beta_{n}\right)\left\|y_{n}-p\right\| \\
& =\left\|y_{n}-p\right\| \\
& \leq\left(1-\alpha_{n}(1-\rho)\right)\left\|x_{n}-p\right\|+\alpha_{n}\|f(p)-p\| \\
& =\left(1-\alpha_{n}(1-\rho)\right)\left\|x_{n}-p\right\|+\alpha_{n}(1-\rho) \frac{\|f(p)-p\|}{1-\rho} \\
& \leq \max \left\{\left\|x_{0}-p\right\|, \frac{\|f(p)-p\|}{1-\rho}\right\} .
\end{aligned}
$$

It immediately follows that $\left\{x_{n}\right\}$ is bounded, and so are the sequences $\left\{y_{n}\right\},\left\{G\left(x_{n}\right)\right\},\left\{G\left(y_{n}\right)\right\}$ due to (4.3) and the nonexpansivity of $G$.

Let us show that $\left\|x_{n+1}-x_{n}\right\| \rightarrow 0$ as $n \rightarrow \infty$. As a matter of fact, from (4.2) we have

$$
\left\{\begin{array}{l}
y_{n}=\alpha_{n} f\left(x_{n}\right)+\left(1-\alpha_{n}\right) S_{n} G\left(x_{n}\right), \\
y_{n-1}=\alpha_{n-1} f\left(x_{n-1}\right)+\left(1-\alpha_{n-1}\right) S_{n-1} G\left(x_{n-1}\right), \quad \forall n \geq 1 .
\end{array}\right.
$$


Simple calculations show that

$$
\begin{aligned}
y_{n}-y_{n-1}= & \alpha_{n}\left(f\left(x_{n}\right)-f\left(x_{n-1}\right)\right)+\left(\alpha_{n}-\alpha_{n-1}\right)\left(f\left(x_{n-1}\right)-S_{n-1} G\left(x_{n-1}\right)\right) \\
& +\left(1-\alpha_{n}\right)\left(S_{n} G\left(x_{n}\right)-S_{n-1} G\left(x_{n-1}\right)\right) .
\end{aligned}
$$

It follows that

$$
\begin{aligned}
\left\|y_{n}-y_{n-1}\right\| \leq & \alpha_{n}\left\|f\left(x_{n}\right)-f\left(x_{n-1}\right)\right\|+\left|\alpha_{n}-\alpha_{n-1}\right|\left\|f\left(x_{n-1}\right)-S_{n-1} G\left(x_{n-1}\right)\right\| \\
& +\left(1-\alpha_{n}\right)\left\|S_{n} G\left(x_{n}\right)-S_{n-1} G\left(x_{n-1}\right)\right\| \\
\leq & \alpha_{n} \rho\left\|x_{n}-x_{n-1}\right\|+\left|\alpha_{n}-\alpha_{n-1}\right|\left\|f\left(x_{n-1}\right)-S_{n-1} G\left(x_{n-1}\right)\right\| \\
& +\left(1-\alpha_{n}\right)\left(\left\|S_{n} G\left(x_{n}\right)-S_{n} G\left(x_{n-1}\right)\right\|+\left\|S_{n} G\left(x_{n-1}\right)-S_{n-1} G\left(x_{n-1}\right)\right\|\right) \\
\leq & \alpha_{n} \rho\left\|x_{n}-x_{n-1}\right\|+\left|\alpha_{n}-\alpha_{n-1}\right|\left\|f\left(x_{n-1}\right)-S_{n-1} G\left(x_{n-1}\right)\right\| \\
& +\left(1-\alpha_{n}\right)\left(\left\|G\left(x_{n}\right)-G\left(x_{n-1}\right)\right\|+\left\|S_{n} G\left(x_{n-1}\right)-S_{n-1} G\left(x_{n-1}\right)\right\|\right) \\
\leq & \alpha_{n} \rho\left\|x_{n}-x_{n-1}\right\|+\left|\alpha_{n}-\alpha_{n-1}\right|\left\|f\left(x_{n-1}\right)-S_{n-1} G\left(x_{n-1}\right)\right\| \\
& +\left(1-\alpha_{n}\right)\left(\left\|x_{n}-x_{n-1}\right\|+\left\|S_{n} G\left(x_{n-1}\right)-S_{n-1} G\left(x_{n-1}\right)\right\|\right) \\
\leq & \left(1-\alpha_{n}(1-\rho)\right)\left\|x_{n}-x_{n-1}\right\|+\left|\alpha_{n}-\alpha_{n-1}\right|\left\|f\left(x_{n-1}\right)-S_{n-1} G\left(x_{n-1}\right)\right\| \\
& +\left\|S_{n} G\left(x_{n-1}\right)-S_{n-1} G\left(x_{n-1}\right)\right\| .
\end{aligned}
$$

So, we have from (4.4)

$$
\begin{aligned}
& \left\|S_{n} G\left(y_{n}\right)-S_{n-1} G\left(y_{n-1}\right)\right\| \\
& \quad \leq\left\|S_{n} G\left(y_{n}\right)-S_{n} G\left(y_{n-1}\right)\right\|+\left\|S_{n} G\left(y_{n-1}\right)-S_{n-1} G\left(y_{n-1}\right)\right\| \\
& \quad \leq\left\|G\left(y_{n}\right)-G\left(y_{n-1}\right)\right\|+\left\|S_{n} G\left(y_{n-1}\right)-S_{n-1} G\left(y_{n-1}\right)\right\| \\
& \quad \leq\left\|y_{n}-y_{n-1}\right\|+\left\|S_{n} G\left(y_{n-1}\right)-S_{n-1} G\left(y_{n-1}\right)\right\| .
\end{aligned}
$$

On the other hand, from (4.2) we have

$$
\left\{\begin{array}{l}
x_{n+1}=\beta_{n} y_{n}+\left(1-\beta_{n}\right) S_{n} G\left(y_{n}\right), \\
x_{n}=\beta_{n-1} y_{n-1}+\left(1-\beta_{n-1}\right) S_{n-1} G\left(y_{n-1}\right) .
\end{array}\right.
$$

Also, simple calculations show that

$$
\begin{aligned}
x_{n+1}-x_{n}= & \beta_{n}\left(y_{n}-y_{n-1}\right)+\left(\beta_{n}-\beta_{n-1}\right)\left(y_{n-1}-S_{n-1} G\left(y_{n-1}\right)\right) \\
& +\left(1-\beta_{n}\right)\left(S_{n} G\left(y_{n}\right)-S_{n-1} G\left(y_{n-1}\right)\right) .
\end{aligned}
$$

Thus, it follows from (4.4)-(4.6) that for all $n \geq 1$

$$
\begin{aligned}
& \left\|x_{n+1}-x_{n}\right\| \\
& \quad \leq \beta_{n}\left\|y_{n}-y_{n-1}\right\|+\left|\beta_{n}-\beta_{n-1}\right|\left\|y_{n-1}-S_{n-1} G\left(y_{n-1}\right)\right\|
\end{aligned}
$$




$$
\begin{aligned}
& +\left(1-\beta_{n}\right)\left\|S_{n} G\left(y_{n}\right)-S_{n-1} G\left(y_{n-1}\right)\right\| \\
\leq & \beta_{n}\left\|y_{n}-y_{n-1}\right\|+\left|\beta_{n}-\beta_{n-1}\right|\left\|y_{n-1}-S_{n-1} G\left(y_{n-1}\right)\right\| \\
& +\left(1-\beta_{n}\right)\left(\left\|y_{n}-y_{n-1}\right\|+\left\|S_{n} G\left(y_{n-1}\right)-S_{n-1} G\left(y_{n-1}\right)\right\|\right) \\
\leq & \left\|y_{n}-y_{n-1}\right\|+\left|\beta_{n}-\beta_{n-1}\right|\left\|y_{n-1}-S_{n-1} G\left(y_{n-1}\right)\right\| \\
& +\left\|S_{n} G\left(y_{n-1}\right)-S_{n-1} G\left(y_{n-1}\right)\right\| \\
\leq & \left(1-\alpha_{n}(1-\rho)\right)\left\|x_{n}-x_{n-1}\right\|+\left|\alpha_{n}-\alpha_{n-1}\right|\left\|f\left(x_{n-1}\right)-S_{n-1} G\left(x_{n-1}\right)\right\| \\
& +\left\|S_{n} G\left(x_{n-1}\right)-S_{n-1} G\left(x_{n-1}\right)\right\|+\left|\beta_{n}-\beta_{n-1}\right|\left\|y_{n-1}-S_{n-1} G\left(y_{n-1}\right)\right\| \\
& +\left\|S_{n} G\left(y_{n-1}\right)-S_{n-1} G\left(y_{n-1}\right)\right\| \\
\leq & \left(1-\alpha_{n}(1-\rho)\right)\left\|x_{n}-x_{n-1}\right\|+M\left(\left|\alpha_{n}-\alpha_{n-1}\right|+\left|\beta_{n}-\beta_{n-1}\right|\right) \\
& +\left\|S_{n} G\left(x_{n-1}\right)-S_{n-1} G\left(x_{n-1}\right)\right\|+\left\|S_{n} G\left(y_{n-1}\right)-S_{n-1} G\left(y_{n-1}\right)\right\|,
\end{aligned}
$$

where $\sup _{n \geq 0}\left\{\left\|f\left(x_{n}\right)-S_{n} G\left(x_{n}\right)\right\|+\left\|y_{n}-S_{n} G\left(y_{n}\right)\right\|\right\} \leq M$ for some $M>0$. Utilizing Lemma 2.2, we deduce from conditions (i), (iii), (iv) and the assumption on $\left\{S_{n}\right\}$ that

$$
\limsup _{n \rightarrow \infty}\left\|x_{n+1}-x_{n}\right\|=0
$$

In terms of (4.4), we also have that $\left\|y_{n}-y_{n-1}\right\| \rightarrow 0$ as $n \rightarrow \infty$.

Let us show that $\left\|x_{n}-y_{n}\right\|$ and $\left\|x_{n}-S_{n} G\left(x_{n}\right)\right\| \rightarrow 0$ as $n \rightarrow \infty$. Indeed, since $y_{n}=\alpha_{n} f\left(x_{n}\right)+$ $\left(1-\alpha_{n}\right) S_{n} G\left(x_{n}\right)$, we get

$$
\lim _{n \rightarrow \infty}\left(1-\alpha_{n}\right)\left\|S_{n} G\left(x_{n}\right)-y_{n}\right\|=\lim _{n \rightarrow \infty} \alpha_{n}\left\|f\left(x_{n}\right)-y_{n}\right\|=0
$$

which together with $\alpha_{n} \rightarrow 0$ implies that

$$
\lim _{n \rightarrow \infty}\left\|S_{n} G\left(x_{n}\right)-y_{n}\right\|=0
$$

Observe that

$$
\begin{aligned}
\left\|x_{n+1}-y_{n}\right\| & =\left(1-\beta_{n}\right)\left\|S_{n} G\left(y_{n}\right)-y_{n}\right\| \\
& \leq\left(1-\beta_{n}\right)\left(\left\|S_{n} G\left(y_{n}\right)-S_{n} G\left(x_{n}\right)\right\|+\left\|S_{n} G\left(x_{n}\right)-y_{n}\right\|\right) \\
& \leq\left(1-\beta_{n}\right)\left(\left\|G\left(y_{n}\right)-G\left(x_{n}\right)\right\|+\left\|S_{n} G\left(x_{n}\right)-y_{n}\right\|\right) \\
& \leq\left(1-\beta_{n}\right)\left(\left\|y_{n}-x_{n}\right\|+\left\|S_{n} G\left(x_{n}\right)-y_{n}\right\|\right) \\
& \leq\left(1-\beta_{n}\right)\left(\left\|y_{n}-x_{n+1}\right\|+\left\|x_{n+1}-x_{n}\right\|+\left\|S_{n} G\left(x_{n}\right)-y_{n}\right\|\right),
\end{aligned}
$$

which together with condition (ii) implies that

$$
\begin{aligned}
\left\|x_{n+1}-y_{n}\right\| & \leq \frac{1-\beta_{n}}{\beta_{n}}\left(\left\|x_{n+1}-x_{n}\right\|+\left\|S_{n} G\left(x_{n}\right)-y_{n}\right\|\right) \\
& \leq \frac{1-a}{a}\left(\left\|x_{n+1}-x_{n}\right\|+\left\|S_{n} G\left(x_{n}\right)-y_{n}\right\|\right) .
\end{aligned}
$$


Obviously, from (4.7) and (4.8) we know that $\left\|x_{n+1}-y_{n}\right\| \rightarrow 0$ as $n \rightarrow \infty$. This implies that

$$
\left\|x_{n}-y_{n}\right\| \leq\left\|x_{n}-x_{n+1}\right\|+\left\|x_{n+1}-y_{n}\right\| \rightarrow 0 \quad \text { as } n \rightarrow \infty
$$

Also, from (4.8) and (4.9) we have

$$
\left\|x_{n}-S_{n} G\left(x_{n}\right)\right\| \leq\left\|x_{n}-y_{n}\right\|+\left\|y_{n}-S_{n} G\left(x_{n}\right)\right\| \rightarrow 0 \quad \text { as } n \rightarrow \infty
$$

Let us show that $\left\|x_{n}-G\left(x_{n}\right)\right\| \rightarrow 0$ as $n \rightarrow \infty$. Indeed, for simplicity, put $q=\Pi_{C}(p-$ $\left.\mu_{2} B_{2} p\right), u_{n}=\Pi_{C}\left(x_{n}-\mu_{2} B_{2} x_{n}\right)$ and $v_{n}=\Pi_{C}\left(u_{n}-\mu_{1} B_{1} u_{n}\right)$. Then $v_{n}=G\left(x_{n}\right)$. From Lemma 2.11 we have

$$
\begin{aligned}
\left\|u_{n}-q\right\|^{2} & =\left\|\Pi_{C}\left(x_{n}-\mu_{2} B_{2} x_{n}\right)-\Pi_{C}\left(p-\mu_{2} B_{2} p\right)\right\|^{2} \\
& \leq\left\|x_{n}-p-\mu_{2}\left(B_{2} x_{n}-B_{2} p\right)\right\|^{2} \\
& \leq\left\|x_{n}-p\right\|^{2}-2 \mu_{2}\left(\alpha_{2}-\kappa^{2} \mu_{2}\right)\left\|B_{2} x_{n}-B_{2} p\right\|^{2},
\end{aligned}
$$

and

$$
\begin{aligned}
\left\|v_{n}-p\right\|^{2} & =\left\|\Pi_{C}\left(u_{n}-\mu_{1} B_{1} u_{n}\right)-\Pi_{C}\left(q-\mu_{1} B_{1} q\right)\right\|^{2} \\
& \leq\left\|u_{n}-q-\mu_{1}\left(B_{1} u_{n}-B_{1} q\right)\right\|^{2} \\
& \leq\left\|u_{n}-q\right\|^{2}-2 \mu_{1}\left(\alpha_{1}-\kappa^{2} \mu_{1}\right)\left\|B_{1} u_{n}-B_{1} q\right\|^{2} .
\end{aligned}
$$

Substituting (4.11) into (4.12), we obtain

$$
\begin{gathered}
\left\|v_{n}-p\right\|^{2} \leq \\
\left\|x_{n}-p\right\|^{2}-2 \mu_{2}\left(\alpha_{2}-\kappa^{2} \mu_{2}\right)\left\|B_{2} x_{n}-B_{2} p\right\|^{2} \\
-2 \mu_{1}\left(\alpha_{1}-\kappa^{2} \mu_{1}\right)\left\|B_{1} u_{n}-B_{1} q\right\|^{2} .
\end{gathered}
$$

According to Lemma 2.2, we have from (4.2)

$$
\begin{aligned}
\left\|y_{n}-p\right\|^{2} & =\left\|\alpha_{n}\left(f\left(x_{n}\right)-f(p)\right)+\left(1-\alpha_{n}\right)\left(S_{n} v_{n}-p\right)+\alpha_{n}(f(p)-p)\right\|^{2} \\
& \leq\left\|\alpha_{n}\left(f\left(x_{n}\right)-f(p)\right)+\left(1-\alpha_{n}\right)\left(S_{n} v_{n}-p\right)\right\|^{2}+2 \alpha_{n}\left\langle f(p)-p, J\left(y_{n}-p\right)\right\rangle \\
& \leq \alpha_{n}\left\|f\left(x_{n}\right)-f(p)\right\|^{2}+\left(1-\alpha_{n}\right)\left\|S_{n} v_{n}-p\right\|^{2}+2 \alpha_{n}\left\langle f(p)-p, J\left(y_{n}-p\right)\right\rangle \\
& \leq \alpha_{n} \rho^{2}\left\|x_{n}-p\right\|^{2}+\left(1-\alpha_{n}\right)\left\|v_{n}-p\right\|^{2}+2 \alpha_{n}\left\langle f(p)-p, J\left(y_{n}-p\right)\right\rangle \\
& \leq \alpha_{n} \rho\left\|x_{n}-p\right\|^{2}+\left(1-\alpha_{n}\right)\left\|v_{n}-p\right\|^{2}+2 \alpha_{n}\|f(p)-p\|\left\|y_{n}-p\right\|,
\end{aligned}
$$

which together with (4.13) and the convexity of $\|\cdot\|^{2}$ implies that

$$
\begin{aligned}
& \left\|x_{n+1}-p\right\|^{2} \\
& \quad=\left\|\beta_{n}\left(y_{n}-p\right)+\left(1-\beta_{n}\right)\left(S_{n} G\left(y_{n}\right)-p\right)\right\|^{2} \\
& \quad \leq \beta_{n}\left\|y_{n}-p\right\|^{2}+\left(1-\beta_{n}\right)\left\|S_{n} G\left(y_{n}\right)-p\right\|^{2} \\
& \quad \leq \beta_{n}\left\|y_{n}-p\right\|^{2}+\left(1-\beta_{n}\right)\left\|G\left(y_{n}\right)-p\right\|^{2}
\end{aligned}
$$




$$
\begin{aligned}
\leq & \beta_{n}\left\|y_{n}-p\right\|^{2}+\left(1-\beta_{n}\right)\left\|y_{n}-p\right\|^{2} \\
= & \left\|y_{n}-p\right\|^{2} \\
\leq & \alpha_{n} \rho\left\|x_{n}-p\right\|^{2}+\left(1-\alpha_{n}\right)\left\|v_{n}-p\right\|^{2}+2 \alpha_{n}\|f(p)-p\|\left\|y_{n}-p\right\| \\
\leq & \alpha_{n} \rho\left\|x_{n}-p\right\|^{2}+\left(1-\alpha_{n}\right)\left\|v_{n}-p\right\|^{2}+M_{1} \alpha_{n} \\
\leq & \alpha_{n} \rho\left\|x_{n}-p\right\|^{2}+\left(1-\alpha_{n}\right)\left[\left\|x_{n}-p\right\|^{2}-2 \mu_{2}\left(\alpha_{2}-\kappa^{2} \mu_{2}\right)\left\|B_{2} x_{n}-B_{2} p\right\|^{2}\right. \\
& \left.-2 \mu_{1}\left(\alpha_{1}-\kappa^{2} \mu_{1}\right)\left\|B_{1} u_{n}-B_{1} q\right\|^{2}\right]+M_{1} \alpha_{n} \\
= & \left(1-\alpha_{n}(1-\rho)\right)\left\|x_{n}-p\right\|^{2}-2\left(1-\alpha_{n}\right)\left[\mu_{2}\left(\alpha_{2}-\kappa^{2} \mu_{2}\right)\left\|B_{2} x_{n}-B_{2} p\right\|^{2}\right. \\
& \left.+\mu_{1}\left(\alpha_{1}-\kappa^{2} \mu_{1}\right)\left\|B_{1} u_{n}-B_{1} q\right\|^{2}\right]+M_{1} \alpha_{n} \\
\leq & \left\|x_{n}-p\right\|^{2}-2\left(1-\alpha_{n}\right)\left[\mu_{2}\left(\alpha_{2}-\kappa^{2} \mu_{2}\right)\left\|B_{2} x_{n}-B_{2} p\right\|^{2}\right. \\
& \left.+\mu_{1}\left(\alpha_{1}-\kappa^{2} \mu_{1}\right)\left\|B_{1} u_{n}-B_{1} q\right\|^{2}\right]+M_{1} \alpha_{n},
\end{aligned}
$$

where $\sup _{n \geq 0}\left\{2\|f(p)-p\|\left\|y_{n}-p\right\|\right\} \leq M_{1}$ for some $M_{1}>0$. So, it follows that

$$
\begin{aligned}
& 2\left(1-\alpha_{n}\right)\left[\mu_{2}\left(\alpha_{2}-\kappa^{2} \mu_{2}\right)\left\|B_{2} x_{n}-B_{2} p\right\|^{2}+\mu_{1}\left(\alpha_{1}-\kappa^{2} \mu_{1}\right)\left\|B_{1} u_{n}-B_{1} q\right\|^{2}\right] \\
& \quad \leq\left\|x_{n}-p\right\|^{2}-\left\|x_{n+1}-p\right\|^{2}+M_{1} \alpha_{n} \\
& \quad \leq\left(\left\|x_{n}-p\right\|+\left\|x_{n+1}-p\right\|\right)\left\|x_{n}-x_{n+1}\right\|+M_{1} \alpha_{n} .
\end{aligned}
$$

Since $0<\mu_{i}<\frac{\alpha_{i}}{\kappa^{2}}$ for $i=1,2$, from (4.7) and $\alpha_{n} \rightarrow 0$ we obtain

$$
\lim _{n \rightarrow \infty}\left\|B_{2} x_{n}-B_{2} p\right\|=0 \text { and } \quad \lim _{n \rightarrow \infty}\left\|B_{1} u_{n}-B_{1} q\right\|=0
$$

Utilizing Proposition 2.1 and Lemma 2.1, we have

$$
\begin{aligned}
\left\|u_{n}-q\right\|^{2}= & \left\|\Pi_{C}\left(x_{n}-\mu_{2} B_{2} x_{n}\right)-\Pi_{C}\left(p-\mu_{2} B_{2} p\right)\right\|^{2} \\
\leq & \left\langle x_{n}-\mu_{2} B_{2} x_{n}-\left(p-\mu_{2} B_{2} p\right), J\left(u_{n}-q\right)\right\rangle \\
= & \left\langle x_{n}-p, J\left(u_{n}-q\right)\right\rangle+\mu_{2}\left\langle B_{2} p-B_{2} x_{n}, J\left(u_{n}-q\right)\right\rangle \\
\leq & \frac{1}{2}\left[\left\|x_{n}-p\right\|^{2}+\left\|u_{n}-q\right\|^{2}-g_{1}\left(\left\|x_{n}-u_{n}-(p-q)\right\|\right)\right] \\
& +\mu_{2}\left\|B_{2} p-B_{2} x_{n}\right\|\left\|u_{n}-q\right\|,
\end{aligned}
$$

which implies that

$$
\left\|u_{n}-q\right\|^{2} \leq\left\|x_{n}-p\right\|^{2}-g_{1}\left(\left\|x_{n}-u_{n}-(p-q)\right\|\right)+2 \mu_{2}\left\|B_{2} p-B_{2} x_{n}\right\|\left\|u_{n}-q\right\| .
$$

In the same way, we derive

$$
\begin{aligned}
\left\|v_{n}-p\right\|^{2} & =\left\|\Pi_{C}\left(u_{n}-\mu_{1} B_{1} u_{n}\right)-\Pi_{C}\left(q-\mu_{1} B_{1} q\right)\right\|^{2} \\
& \leq\left\langle u_{n}-\mu_{1} B_{1} u_{n}-\left(q-\mu_{1} B_{1} q\right), J\left(v_{n}-p\right)\right\rangle \\
& =\left\langle u_{n}-q, J\left(v_{n}-p\right)\right\rangle+\mu_{1}\left\langle B_{1} q-B_{1} u_{n}, J\left(v_{n}-p\right)\right\rangle
\end{aligned}
$$




$$
\begin{aligned}
\leq & \frac{1}{2}\left[\left\|u_{n}-q\right\|^{2}+\left\|v_{n}-p\right\|^{2}-g_{2}\left(\left\|u_{n}-v_{n}+(p-q)\right\|\right)\right] \\
& +\mu_{1}\left\|B_{1} q-B_{1} u_{n}\right\|\left\|v_{n}-p\right\|,
\end{aligned}
$$

which implies that

$$
\left\|v_{n}-p\right\|^{2} \leq\left\|u_{n}-q\right\|^{2}-g_{2}\left(\left\|u_{n}-v_{n}+(p-q)\right\|\right)+2 \mu_{1}\left\|B_{1} q-B_{1} u_{n}\right\|\left\|v_{n}-p\right\| .
$$

Substituting (4.16) into (4.17), we get

$$
\begin{aligned}
\left\|v_{n}-p\right\|^{2} \leq & \left\|x_{n}-p\right\|^{2}-g_{1}\left(\left\|x_{n}-u_{n}-(p-q)\right\|\right)-g_{2}\left(\left\|u_{n}-v_{n}+(p-q)\right\|\right) \\
& +2 \mu_{2}\left\|B_{2} p-B_{2} x_{n}\right\|\left\|u_{n}-q\right\|+2 \mu_{1}\left\|B_{1} q-B_{1} u_{n}\right\|\left\|v_{n}-p\right\| .
\end{aligned}
$$

From (4.14) and (4.18), we have

$$
\begin{aligned}
&\left\|x_{n+1}-p\right\|^{2} \\
& \leq \alpha_{n} \rho\left\|x_{n}-p\right\|^{2}+\left(1-\alpha_{n}\right)\left\|v_{n}-p\right\|^{2}+M_{1} \alpha_{n} \\
& \leq \alpha_{n} \rho\left\|x_{n}-p\right\|^{2}+\left(1-\alpha_{n}\right)\left[\left\|x_{n}-p\right\|^{2}-g_{1}\left(\left\|x_{n}-u_{n}-(p-q)\right\|\right)\right. \\
& \quad-g_{2}\left(\left\|u_{n}-v_{n}+(p-q)\right\|\right)+2 \mu_{2}\left\|B_{2} p-B_{2} x_{n}\right\|\left\|u_{n}-q\right\| \\
&\left.+2 \mu_{1}\left\|B_{1} q-B_{1} u_{n}\right\|\left\|v_{n}-p\right\|\right]+M_{1} \alpha_{n} \\
& \leq\left(1-\alpha_{n}(1-\rho)\right)\left\|x_{n}-p\right\|^{2}-\left(1-\alpha_{n}\right)\left[g_{1}\left(\left\|x_{n}-u_{n}-(p-q)\right\|\right)\right. \\
&\left.+g_{2}\left(\left\|u_{n}-v_{n}+(p-q)\right\|\right)\right]+2 \mu_{2}\left\|B_{2} p-B_{2} x_{n}\right\|\left\|u_{n}-q\right\| \\
&+2 \mu_{1}\left\|B_{1} q-B_{1} u_{n}\right\|\left\|v_{n}-p\right\|+M_{1} \alpha_{n} \\
& \leq\left\|x_{n}-p\right\|^{2}-\left(1-\alpha_{n}\right)\left[g_{1}\left(\left\|x_{n}-u_{n}-(p-q)\right\|\right)+g_{2}\left(\left\|u_{n}-v_{n}+(p-q)\right\|\right)\right] \\
&+2 \mu_{2}\left\|B_{2} p-B_{2} x_{n}\right\|\left\|u_{n}-q\right\|+2 \mu_{1}\left\|B_{1} q-B_{1} u_{n}\right\|\left\|v_{n}-p\right\|+M_{1} \alpha_{n},
\end{aligned}
$$

which hence implies that

$$
\begin{aligned}
\left(1-\alpha_{n}\right) & {\left[g_{1}\left(\left\|x_{n}-u_{n}-(p-q)\right\|\right)+g_{2}\left(\left\|u_{n}-v_{n}+(p-q)\right\|\right)\right] } \\
\leq & \left\|x_{n}-p\right\|^{2}-\left\|x_{n+1}-p\right\|^{2}+2 \mu_{2}\left\|B_{2} p-B_{2} x_{n}\right\|\left\|u_{n}-q\right\| \\
& \quad+2 \mu_{1}\left\|B_{1} q-B_{1} u_{n}\right\|\left\|v_{n}-p\right\|+M_{1} \alpha_{n} \\
\leq & \alpha_{n} M_{1}+\left(\left\|x_{n}-p\right\|+\left\|x_{n+1}-p\right\|\right)\left\|x_{n}-x_{n+1}\right\|+2 \mu_{2}\left\|B_{2} p-B_{2} x_{n}\right\|\left\|u_{n}-q\right\| \\
& +2 \mu_{1}\left\|B_{1} q-B_{1} u_{n}\right\|\left\|v_{n}-p\right\| .
\end{aligned}
$$

Thus, from (4.7), (4.15) and $\alpha_{n} \rightarrow 0$ we have

$$
\lim _{n \rightarrow \infty} g_{1}\left(\left\|x_{n}-u_{n}-(p-q)\right\|\right)=0 \quad \text { and } \quad \lim _{n \rightarrow \infty} g_{2}\left(\left\|u_{n}-v_{n}+(p-q)\right\|\right)=0 .
$$

Utilizing the properties of $g_{1}$ and $g_{2}$, we deduce that

$$
\lim _{n \rightarrow \infty}\left\|x_{n}-u_{n}-(p-q)\right\|=0 \quad \text { and } \quad \lim _{n \rightarrow \infty}\left\|u_{n}-v_{n}+(p-q)\right\|=0 .
$$


From (4.20), we obtain

$$
\left\|x_{n}-v_{n}\right\| \leq\left\|x_{n}-u_{n}-(p-q)\right\|+\left\|u_{n}-v_{n}+(p-q)\right\| \rightarrow 0 \quad \text { as } n \rightarrow \infty \text {. }
$$

That is,

$$
\lim _{n \rightarrow \infty}\left\|x_{n}-G\left(x_{n}\right)\right\|=0
$$

On the other hand, we observe that

$$
\left\|S_{n} G\left(x_{n}\right)-G\left(x_{n}\right)\right\| \leq\left\|S_{n} G\left(x_{n}\right)-y_{n}\right\|+\left\|y_{n}-x_{n}\right\|+\left\|x_{n}-G\left(x_{n}\right)\right\| .
$$

So, it follows from (4.8), (4.9) and (4.21) that

$$
\lim _{n \rightarrow \infty}\left\|S_{n} G\left(x_{n}\right)-G\left(x_{n}\right)\right\|=0 .
$$

By (4.23) and Lemma 2.6, we have

$$
\begin{aligned}
\left\|S G\left(x_{n}\right)-G\left(x_{n}\right)\right\| & \leq\left\|S G\left(x_{n}\right)-S_{n} G\left(x_{n}\right)\right\|+\left\|S_{n} G\left(x_{n}\right)-G\left(x_{n}\right)\right\| \\
& \rightarrow 0 \quad \text { as } n \rightarrow \infty .
\end{aligned}
$$

In terms of (4.21) and (4.24), we have

$$
\begin{aligned}
\left\|x_{n}-S x_{n}\right\| & \leq\left\|x_{n}-G\left(x_{n}\right)\right\|+\left\|G\left(x_{n}\right)-S G\left(x_{n}\right)\right\|+\left\|S G\left(x_{n}\right)-S x_{n}\right\| \\
& \leq 2\left\|x_{n}-G\left(x_{n}\right)\right\|+\left\|G\left(x_{n}\right)-S G\left(x_{n}\right)\right\| \\
& \rightarrow 0 \quad \text { as } n \rightarrow \infty .
\end{aligned}
$$

Define a mapping $W x=(1-\theta) S x+\theta G(x)$ and $\theta \in(0,1)$ is a constant. Then by Lemma 2.8 we have that $\operatorname{Fix}(W)=\operatorname{Fix}(S) \cap \operatorname{Fix}(G)=F$. We observe that

$$
\left\|x_{n}-W x_{n}\right\|=\left\|(1-\theta)\left(x_{n}-S x_{n}\right)+\theta\left(x_{n}-G\left(x_{n}\right)\right)\right\| \leq(1-\theta)\left\|x_{n}-S x_{n}\right\|+\theta\left\|x_{n}-G\left(x_{n}\right)\right\| .
$$

From (4.21) and (4.25), we obtain

$$
\lim _{n \rightarrow \infty}\left\|x_{n}-W x_{n}\right\|=0
$$

Utilizing the arguments similar to those of (3.29) in the proof of Theorem 3.1, we can deduce that

$$
\limsup _{n \rightarrow \infty}\left\langle f(q)-q, J\left(y_{n}-q\right)\right\rangle \leq 0
$$

Finally, let us show that $x_{n} \rightarrow q$ as $n \rightarrow \infty$. We observe that

$$
\begin{aligned}
& \left\|y_{n}-q\right\|^{2} \\
& \quad=\left\|\alpha_{n}\left(f\left(x_{n}\right)-f(q)\right)+\left(1-\alpha_{n}\right)\left(S_{n} G\left(x_{n}\right)-q\right)+\alpha_{n}(f(q)-q)\right\|^{2}
\end{aligned}
$$




$$
\begin{aligned}
& \leq\left\|\alpha_{n}\left(f\left(x_{n}\right)-f(q)\right)+\left(1-\alpha_{n}\right)\left(S_{n} G\left(x_{n}\right)-q\right)\right\|^{2}+2 \alpha_{n}\left\langle f(q)-q, J\left(y_{n}-q\right)\right\rangle \\
& \leq \alpha_{n}\left\|f\left(x_{n}\right)-f(q)\right\|^{2}+\left(1-\alpha_{n}\right)\left\|S_{n} G\left(x_{n}\right)-q\right\|^{2}+2 \alpha_{n}\left\langle f(q)-q, J\left(y_{n}-q\right)\right\rangle \\
& \leq \alpha_{n} \rho\left\|x_{n}-q\right\|^{2}+\left(1-\alpha_{n}\right)\left\|x_{n}-q\right\|^{2}+2 \alpha_{n}\left\langle f(q)-q, J\left(y_{n}-q\right)\right\rangle \\
& =\left(1-\alpha_{n}(1-\rho)\right)\left\|x_{n}-q\right\|^{2}+2 \alpha_{n}\left\langle f(q)-q, J\left(y_{n}-q\right)\right\rangle .
\end{aligned}
$$

By the convexity of $\|\cdot\|^{2}$ and (4.2), we get

$$
\left\|x_{n+1}-q\right\|^{2} \leq \beta_{n}\left\|y_{n}-q\right\|^{2}+\left(1-\beta_{n}\right)\left\|S_{n} G\left(y_{n}\right)-q\right\|^{2} \leq\left\|y_{n}-q\right\|^{2},
$$

which together with (4.28) leads to

$$
\begin{aligned}
\left\|x_{n+1}-q\right\|^{2} & \leq\left(1-\alpha_{n}(1-\rho)\right)\left\|x_{n}-q\right\|^{2}+2 \alpha_{n}\left\langle f(q)-q, J\left(y_{n}-q\right)\right\rangle \\
& =\left(1-\alpha_{n}(1-\rho)\right)\left\|x_{n}-q\right\|^{2}+\alpha_{n}(1-\rho) \cdot \frac{2\left\langle f(q)-q, J\left(y_{n}-q\right)\right\rangle}{1-\rho} .
\end{aligned}
$$

Applying Lemma 2.2 to (4.29), we obtain that $x_{n} \rightarrow q$ as $n \rightarrow \infty$. This completes the proof.

Corollary 4.1 Let $C$ be a nonempty closed convex subset of a uniformly convex and 2uniformly smooth Banach space $X$. Let $\Pi_{C}$ be a sunny nonexpansive retraction from $X$ onto C. Let the mapping $B_{i}: C \rightarrow X$ be $\alpha_{i}$-inverse-strongly accretive for $i=1,2$. Let $f: C \rightarrow$ $C$ be a contraction with coefficient $\rho \in(0,1)$. Let $S$ be a nonexpansive mapping of $C$ into itself such that $F=\operatorname{Fix}(S) \cap \Omega \neq \emptyset$, where $\Omega$ is a fixed point set of the mapping G. For arbitrarily given $x_{0} \in C$, let $\left\{x_{n}\right\}$ be a sequence generated by

$$
\left\{\begin{array}{l}
y_{n}=\alpha_{n} f\left(x_{n}\right)+\left(1-\alpha_{n}\right) S \Pi_{C}\left(I-\mu_{1} B_{1}\right) \Pi_{C}\left(I-\mu_{2} B_{2}\right) x_{n}, \\
x_{n+1}=\beta_{n} y_{n}+\left(1-\beta_{n}\right) S \Pi_{C}\left(I-\mu_{1} B_{1}\right) \Pi_{C}\left(I-\mu_{2} B_{2}\right) y_{n}, \quad \forall n \geq 0,
\end{array}\right.
$$

where $0<\mu_{i}<\frac{\alpha_{i}}{\kappa^{2}}$ for $i=1,2$. Suppose that $\left\{\alpha_{n}\right\}$ and $\left\{\beta_{n}\right\}$ are sequences in $(0,1]$ satisfying the following conditions:

(i) $\lim _{n \rightarrow \infty} \alpha_{n}=0$ and $\sum_{n=0}^{\infty} \alpha_{n}=\infty$;

(ii) $\left\{\beta_{n}\right\} \subset[a, 1]$ for some $a \in(0,1)$;

(iii) $\sum_{n=1}^{\infty}\left|\alpha_{n}-\alpha_{n-1}\right|<\infty$ or $\lim _{n \rightarrow \infty} \alpha_{n-1} / \alpha_{n}=1$;

(iv) $\sum_{n=1}^{\infty}\left|\beta_{n}-\beta_{n-1}\right|<\infty$ or $\lim _{n \rightarrow \infty}\left|\beta_{n}-\beta_{n-1}\right| / \alpha_{n}=0$.

Then $\left\{x_{n}\right\}$ converges strongly to $q \in F$, which solves the following VIP:

$$
\langle q-f(q), J(q-p)\rangle \leq 0, \quad \forall p \in F
$$

Remark 4.1 Theorem 4.1 improves, extends, supplements and develops [14, Theorem 3.1] in the following aspects. The composite iterative algorithm in [14, Theorem 3.1] is extended to develop the composite iterative algorithm in Theorem 4.1. Compared with the iterative algorithm in [14, Theorem 3.1], each iteration step in the iterative algorithm of Theorem 4.1 is very different from the corresponding step in the iterative algorithm of [14, Theorem 3.1] because each iteration step in the iterative algorithm of Theorem 4.1 involves the composite operator $S_{n} \Pi_{C}\left(I-\mu_{1} B_{1}\right) \Pi_{C}\left(I-\mu_{2} B_{2}\right)$. In the proof of [14, Theorem 3.1], 
Lemma 2.4 was used to derive $\left\|x_{n+1}-x_{n}\right\| \rightarrow 0$. However, in the proof of Theorem 4.1, we only use Lemma 2.2 to derive $\left\|x_{n+1}-x_{n}\right\| \rightarrow 0$. Thus, Theorem 4.1 drops the restriction $\lim \sup _{n \rightarrow \infty} \beta_{n}<1$.

Corollary 4.2 Let $C$ be a nonempty closed convex subset of a real Hilbert space H. Let the mapping $B_{i}: C \rightarrow H$ be $\alpha_{i}$-inverse-strongly monotone for $i=1$, 2. Let $f: C \rightarrow C$ be a contraction with coefficient $\rho \in(0,1)$. Let $\left\{S_{n}\right\}_{n=0}^{\infty}$ be an infinite family of nonexpansive mappings of $C$ into itself such that $F=\bigcap_{i=0}^{\infty} \operatorname{Fix}\left(S_{i}\right) \cap \Omega \neq \emptyset$, where $\Omega$ is a fixed point set of the mapping G. For arbitrarily given $x_{0} \in C$, let $\left\{x_{n}\right\}$ be a sequence generated by

$$
\left\{\begin{array}{l}
y_{n}=\alpha_{n} f\left(x_{n}\right)+\left(1-\alpha_{n}\right) S_{n} P_{C}\left(I-\mu_{1} B_{1}\right) P_{C}\left(I-\mu_{2} B_{2}\right) x_{n}, \\
x_{n+1}=\beta_{n} y_{n}+\left(1-\beta_{n}\right) S_{n} P_{C}\left(I-\mu_{1} B_{1}\right) P_{C}\left(I-\mu_{2} B_{2}\right) y_{n}, \quad \forall n \geq 0,
\end{array}\right.
$$

where $0<\mu_{i}<2 \alpha_{i}$ for $i=1,2$. Suppose that $\left\{\alpha_{n}\right\}$ and $\left\{\beta_{n}\right\}$ are sequences in $(0,1]$ satisfying the following conditions:

(i) $\lim _{n \rightarrow \infty} \alpha_{n}=0$ and $\sum_{n=0}^{\infty} \alpha_{n}=\infty$;

(ii) $\left\{\beta_{n}\right\} \subset[a, 1]$ for some $a \in(0,1)$;

(iii) $\sum_{n=1}^{\infty}\left|\alpha_{n}-\alpha_{n-1}\right|<\infty$ or $\lim _{n \rightarrow \infty} \alpha_{n-1} / \alpha_{n}=1$;

(iv) $\sum_{n=1}^{\infty}\left|\beta_{n}-\beta_{n-1}\right|<\infty$ or $\lim _{n \rightarrow \infty}\left|\beta_{n}-\beta_{n-1}\right| / \alpha_{n}=0$.

Assume that $\sum_{n=1}^{\infty} \sup _{x \in D}\left\|S_{n} x-S_{n-1} x\right\|<\infty$ for any bounded subset $D$ of $C$ and let $S$ be a mapping of $C$ into itself defined by $S x=\lim _{n \rightarrow \infty} S_{n} x$ for all $x \in C$ and suppose that $\operatorname{Fix}(S)=$ $\bigcap_{i=0}^{\infty} \operatorname{Fix}\left(S_{i}\right)$. Then $\left\{x_{n}\right\}$ converges strongly to $q \in F$, which solves the following VIP:

$$
\langle q-f(q), J(q-p)\rangle \leq 0, \quad \forall p \in F
$$

Corollary 4.3 Let $C$ be a nonempty closed convex subset of a real Hilbert space H. Let the mapping $B_{i}: C \rightarrow H$ be $\alpha_{i}$-inverse-strongly monotone for $i=1,2$. Let $f: C \rightarrow C$ be a contraction with coefficient $\rho \in(0,1)$. Let $S$ be a nonexpansive mapping of $C$ into itself such that $F=\operatorname{Fix}(S) \cap \Omega \neq \emptyset$, where $\Omega$ is a fixed point set of the mapping G. For arbitrarily given $x_{0} \in C$, let $\left\{x_{n}\right\}$ be a sequence generated by

$$
\left\{\begin{array}{l}
y_{n}=\alpha_{n} f\left(x_{n}\right)+\left(1-\alpha_{n}\right) S P_{C}\left(I-\mu_{1} B_{1}\right) P_{C}\left(I-\mu_{2} B_{2}\right) x_{n}, \\
x_{n+1}=\beta_{n} y_{n}+\left(1-\beta_{n}\right) S P_{C}\left(I-\mu_{1} B_{1}\right) P_{C}\left(I-\mu_{2} B_{2}\right) y_{n}, \quad \forall n \geq 0,
\end{array}\right.
$$

where $0<\mu_{i}<\frac{\alpha_{i}}{\kappa^{2}}$ for $i=1,2$. Suppose that $\left\{\alpha_{n}\right\}$ and $\left\{\beta_{n}\right\}$ are sequences in $(0,1]$ satisfying the following conditions:

(i) $\lim _{n \rightarrow \infty} \alpha_{n}=0$ and $\sum_{n=0}^{\infty} \alpha_{n}=\infty$;

(ii) $\left\{\beta_{n}\right\} \subset[a, 1]$ for some $a \in(0,1)$;

(iii) $\sum_{n=1}^{\infty}\left|\alpha_{n}-\alpha_{n-1}\right|<\infty$ or $\lim _{n \rightarrow \infty} \alpha_{n-1} / \alpha_{n}=1$;

(iv) $\sum_{n=1}^{\infty}\left|\beta_{n}-\beta_{n-1}\right|<\infty$ or $\lim _{n \rightarrow \infty}\left|\beta_{n}-\beta_{n-1}\right| / \alpha_{n}=0$.

Then $\left\{x_{n}\right\}$ converges strongly to $q \in F$, which solves the following VIP:

$$
\langle q-f(q), q-p\rangle \leq 0, \quad \forall p \in F
$$


Now, we say that a mapping $T: C \rightarrow C$ has property $(*)$ if there exists a constant $k \in[0,1)$ such that

$$
\|T x-T y\|^{2} \leq\|x-y\|^{2}+k\|(I-T) x-(I-T) y\|^{2}, \quad \forall x, y \in C .
$$

Whenever $k=0$, then $T$ is nonexpansive. Put $A=I-T$, where $T: C \rightarrow C$ is a mapping having property $(*)$. Then $A$ is $(1-k) / 2$-inverse-strongly monotone. Indeed, we have

$$
\|(I-A) x-(I-A) y\|^{2} \leq\|x-y\|^{2}+k\|A x-A y\|^{2}, \quad \forall x, y \in C .
$$

Since $H$ is a real Hilbert space, we have

$$
\|(I-A) x-(I-A) y\|^{2}=\|x-y\|^{2}+\|A x-A y\|^{2}-2\langle x-y, A x-A y\rangle,
$$

and hence

$$
\begin{aligned}
& \langle x-y, A x-A y\rangle \geq \frac{1-k}{2}\|A x-A y\|^{2} \\
& \Rightarrow \quad\|(I-T) x-(I-T) y\| \leq \frac{2}{1-k}\|x-y\| \\
& \Rightarrow \quad\|T x-T y\|^{2} \leq\|x-y\|^{2}+k\|(I-T) x-(I-T) y\|^{2} \leq\left(\frac{1+k}{1-k}\right)^{2}\|x-y\|^{2} .
\end{aligned}
$$

Thus, if $T$ is a mapping having property ( $*$ ), then $T$ is Lipschitz continuous with constant $\frac{1+k}{1-k}$, i.e., $\|T x-T y\| \leq \frac{1+k}{1-k}\|x-y\|$ for all $x, y \in C$. We denote by Fix $(T)$ a fixed point set of $T$. It is obvious that the class of mappings having property $(*)$ strictly includes the class of nonexpansive mappings.

Further, utilizing Corollary 4.3 we first derive a strong convergence result for finding a common fixed point of a nonexpansive mapping and a mapping having property $(*)$.

Corollary 4.4 Let $C$ be a nonempty closed convex subset of a real Hilbert space H. Let $T: C \rightarrow C$ be a mapping having property $(*)$ and let $S: C \rightarrow C$ be a nonexpansive mapping such that $\operatorname{Fix}(S) \cap \operatorname{Fix}(T) \neq \emptyset$. Let $f: C \rightarrow C$ be a contraction with coefficient $\rho \in(0,1)$. For arbitrarily given $x_{0} \in C$, let $\left\{x_{n}\right\}$ be a sequence generated by

$$
\left\{\begin{array}{l}
y_{n}=\alpha_{n} f\left(x_{n}\right)+\left(1-\alpha_{n}\right) S\left((1-\lambda) x_{n}+\lambda T x_{n}\right), \\
x_{n+1}=\beta_{n} y_{n}+\left(1-\beta_{n}\right) S\left((1-\lambda) y_{n}+\lambda T y_{n}\right), \quad \forall n \geq 0
\end{array}\right.
$$

where $0<\lambda<1-k$. Suppose that $\left\{\alpha_{n}\right\}$ and $\left\{\beta_{n}\right\}$ are sequences in $(0,1]$ satisfying the following conditions:

(i) $\lim _{n \rightarrow \infty} \alpha_{n}=0$ and $\sum_{n=0}^{\infty} \alpha_{n}=\infty$;

(ii) $\left\{\beta_{n}\right\} \subset[a, 1]$ for some $a \in(0,1)$;

(iii) $\sum_{n=1}^{\infty}\left|\alpha_{n}-\alpha_{n-1}\right|<\infty$ or $\lim _{n \rightarrow \infty} \alpha_{n-1} / \alpha_{n}=1$;

(iv) $\sum_{n=1}^{\infty}\left|\beta_{n}-\beta_{n-1}\right|<\infty$ or $\lim _{n \rightarrow \infty}\left|\beta_{n}-\beta_{n-1}\right| / \alpha_{n}=0$.

Then $\left\{x_{n}\right\}$ converges strongly to $q \in \operatorname{Fix}(S) \cap \operatorname{Fix}(T)$, which solves the following VIP:

$$
\langle q-f(q), q-p\rangle \leq 0, \quad \forall p \in \operatorname{Fix}(S) \cap \operatorname{Fix}(T) .
$$


Proof In Corollary 4.3, we put $B_{1}=I-T, B_{2}=0$ and $\mu_{1}=\lambda$. Then GSVI (1.1) is equivalent to the VIP of finding $x^{*} \in C$ such that

$$
\left\langle B_{1} x^{*}, x-x^{*}\right\rangle \geq 0, \quad \forall x \in C .
$$

In this case, $B_{1}$ is $(1-k) / 2$-inverse-strongly monotone. It is not hard to see that $\operatorname{Fix}(T)=$ $\mathrm{VI}\left(C, B_{1}\right)$. As a matter of fact, we have, for $\lambda>0$,

$$
\begin{aligned}
u \in \operatorname{VI}\left(C, B_{1}\right) & \Leftrightarrow \quad\left\langle B_{1} u, y-u\right\rangle \geq 0 \quad \forall y \in C \\
& \Leftrightarrow \quad\left\langle u-\lambda B_{1} u-u, u-y\right\rangle \geq 0 \quad \forall y \in C \\
& \Leftrightarrow \quad u=P_{C}\left(u-\lambda B_{1} u\right) \\
& \Leftrightarrow \quad u=P_{C}(u-\lambda u+\lambda T u) \\
& \Leftrightarrow \quad\langle u-\lambda u+\lambda T u-u, u-y\rangle \geq 0 \quad \forall y \in C \\
& \Leftrightarrow \quad\langle u-T u, u-y\rangle \leq 0 \quad \forall y \in C \\
& \Leftrightarrow u=T u \\
& \Leftrightarrow u \in \operatorname{Fix}(T) .
\end{aligned}
$$

Accordingly, we know that $F=\operatorname{Fix}(S) \cap \Omega=\operatorname{Fix}(S) \cap \operatorname{Fix}(T)$,

$$
\begin{aligned}
P_{C}\left(I-\mu_{1} B_{1}\right) P_{C}\left(I-\mu_{2} B_{2}\right) x_{n} & =P_{C}\left(I-\mu_{1} B_{1}\right) x_{n} \\
& =P_{C}\left((1-\lambda) x_{n}+\lambda T x_{n}\right)=(1-\lambda) x_{n}+\lambda T x_{n},
\end{aligned}
$$

and

$$
\begin{aligned}
P_{C}\left(I-\mu_{1} B_{1}\right) P_{C}\left(I-\mu_{2} B_{2}\right) y_{n} & =P_{C}\left(I-\mu_{1} B_{1}\right) y_{n} \\
& =P_{C}\left((1-\lambda) y_{n}+\lambda T y_{n}\right)=(1-\lambda) y_{n}+\lambda T y_{n} .
\end{aligned}
$$

So, scheme (4.2) reduces to (4.30). Therefore, the desired result follows from Corollary 4.3.

Utilizing Corollary 4.3, we also have the following result.

Corollary 4.5 Let $H$ be a real Hilbert space. Let $A$ be an $\alpha$-inverse-strongly monotone mapping of $H$ into itself and let $S$ be a nonexpansive mapping of $H$ into itself such that $\operatorname{Fix}(S) \cap \cap A^{-1} 0 \neq \emptyset$. Let $f: H \rightarrow H$ be a contraction with coefficient $\rho \in(0,1)$. For arbitrarily given $x_{0} \in H$, let $\left\{x_{n}\right\}$ be a sequence generated by

$$
\left\{\begin{array}{l}
y_{n}=\alpha_{n} f\left(x_{n}\right)+\left(1-\alpha_{n}\right) S\left(x_{n}-\lambda A x_{n}\right), \\
x_{n+1}=\beta_{n} y_{n}+\left(1-\beta_{n}\right) S\left(y_{n}-\lambda A y_{n}\right), \quad \forall n \geq 0,
\end{array}\right.
$$

where $0<\lambda<2 \alpha$. Suppose that $\left\{\alpha_{n}\right\}$ and $\left\{\beta_{n}\right\}$ are sequences in $(0,1]$ satisfying the following conditions: 
(i) $\lim _{n \rightarrow \infty} \alpha_{n}=0$ and $\sum_{n=0}^{\infty} \alpha_{n}=\infty$;

(ii) $\left\{\beta_{n}\right\} \subset[a, 1]$ for some $a \in(0,1)$;

(iii) $\sum_{n=1}^{\infty}\left|\alpha_{n}-\alpha_{n-1}\right|<\infty$ or $\lim _{n \rightarrow \infty} \alpha_{n-1} / \alpha_{n}=1$;

(iv) $\sum_{n=1}^{\infty}\left|\beta_{n}-\beta_{n-1}\right|<\infty$ or $\lim _{n \rightarrow \infty}\left|\beta_{n}-\beta_{n-1}\right| / \alpha_{n}=0$.

Then $\left\{x_{n}\right\}$ converges strongly to $q \in \operatorname{Fix}(S) \cap A^{-1} 0$, which solves the following VIP:

$$
\langle q-f(q), q-p\rangle \leq 0, \quad \forall p \in \operatorname{Fix}(S) \cap A^{-1} 0 .
$$

Proof In Corollary 4.3, we put $C=H, B_{1}=A, B_{2}=0$ and $\mu_{1}=\lambda$. Then we know that $P_{H}=I$ and $A^{-1} 0=\operatorname{VI}(H, A)=\Omega$. Moreover, we know that $F=\operatorname{Fix}(S) \cap \Omega=\operatorname{Fix}(S) \cap A^{-1} 0$,

$$
P_{C}\left(I-\mu_{1} B_{1}\right) P_{C}\left(I-\mu_{2} B_{2}\right) x_{n}=x_{n}-\lambda A x_{n},
$$

and

$$
P_{C}\left(I-\mu_{1} B_{1}\right) P_{C}\left(I-\mu_{2} B_{2}\right) y_{n}=y_{n}-\lambda A y_{n} .
$$

So, scheme (4.2) reduces to (4.31). Therefore, the desired result follows from Corollary 4.3.

\section{Competing interests}

The authors declare that they have no competing interests.

\section{Authors' contributions}

All authors participated in the design of this work and performed equally. All authors read and approved the final manuscript.

\section{Author details}

'Department of Mathematics, Shanghai Normal University, and Scientific Computing Key Laboratory of Shanghai Universities, Shanghai, 200234, China. '2Department of Mathematics, King Abdulaziz University, P.O. Box 80203, Jeddah, 21589, Saudi Arabia. ${ }^{3}$ Center for Fundamental Science, Kaohsiung Medical University, Kaohsiung, 807, Taiwan.

\section{Acknowledgements}

This article was partially funded by the Deanship of Scientific Research (DSR), King Abdulaziz University. Therefore, the second and third authors gratefully acknowledge with thanks DSR for financial support. This research was partially supported to the first author by the National Science Foundation of China (11071169), Innovation Program of Shanghai Municipal Education Commission (09ZZ133) and Ph.D. Program Foundation of Ministry of Education of China (20123127110002). The authors thank the referees for their valuable comments and suggestions.

Received: 10 April 2013 Accepted: 17 June 2013 Published: 3 July 2013

\section{References}

1. Aubin, JP: Mathematical Models of Game and Economic Theory. North-Holland, Amsterdam (1979)

2. Facchinei, F, Pang, JS: Finite-Dimensional Variational Inequalities and Complementarity Problems, vol. 1. Springer, New York (2003)

3. Facchinei, F, Pang, JS: Finite-Dimensional Variational Inequalities and Complementarity Problems, vol. 2. Springer, New York (2003)

4. Ansari, QH, Schaible, S, Yao, JC: System of vector equilibrium problems and its applications. J. Optim. Theory Appl. 107(3), 547-557 (2000)

5. Ansari, QH, Yao, JC: A fixed point theorem and its applications to the system of variational inequalities. Bull. Aust. Math. Soc. 59, 433-442 (1999)

6. Ansari, QH, Yao, JC: Systems of generalized variational inequalities and their applications. Appl. Anal. 76(3-4), 203-217 (2000)

7. Ansari, QH, Khan, Z: Relatively B-pseudomonotone variational inequalities over product of sets. JIPAM. J. Inequal. Pure Appl. Math. 4(1), Article ID 6 (2003)

8. Ansari, QH, Khan, Z: Densely relative pseudomonotone variational inequalities over product of sets. J. Nonlinear Convex Anal. 7(2), 179-188 (2006)

9. Cai, G, Bu, SQ: Convergence analysis for variational inequality problems and fixed point problems in 2-uniformly smooth and uniformly convex Banach spaces. Math. Comput. Model. 55, 538-546 (2012) 
10. Korpelevich, GM: An extragradient method for finding saddle points and for other problems. Ėkon. Mat. Metody 12, 747-756 (1976)

11. Reich, S: Weak convergence theorems for nonexpansive mappings in Banach spaces. J. Math. Anal. Appl. 67, 274-276 (1979)

12. Xu, HK: Iterative algorithms for nonlinear operators. J. Lond. Math. Soc. 66, 240-256 (2002)

13. Suzuki, T: Strong convergence of Krasnoselskii and Mann's type sequences for one parameter nonexpansive semigroups without Bochner integrals. J. Math. Anal. Appl. 305, 227-239 (2005)

14. Xu, HK: Inequalities in Banach spaces with applications. Nonlinear Anal. 16, 1127-1138 (1991)

15. Aoyama, K, Kimura, Y, Takahashi, W, Toyoda, M: Approximation of common fixed points of a countable family of nonexpansive mappings in a Banach space. Nonlinear Anal. 67, 2350-2360 (2007)

16. Jung, JS: Iterative approaches to common fixed points of nonexpansive mappings in Banach spaces. J. Math. Anal. Appl. 302, 509-520 (2005)

17. Bruck, RE: Properties of fixed point sets of nonexpansive mappings in Banach spaces. Trans. Am. Math. Soc. 179 251-262 (1973)

18. Kamimura, S, Takahashi, W: Strong convergence of a proximal-type algorithm in a Banach space. SIAM J. Optim. 13, 938-945 (2002)

doi:10.1186/1687-1812-2013-176

Cite this article as: Ceng et al.: On solutions of a system of variational inequalities and fixed point problems in Banach spaces. Fixed Point Theory and Applications 2013 2013:176.

\section{Submit your manuscript to a SpringerOpen ${ }^{0}$ journal and benefit from:}

- Convenient online submission

- Rigorous peer review

- Immediate publication on acceptance

- Open access: articles freely available online

- High visibility within the field

- Retaining the copyright to your article 